\title{
A SPECTRAL METHOD FOR NUMERICAL ELASTODYNAMIC FRACTURE ANALYSIS WITHOUT SPATIAL REPLICATION OF THE RUPTURE EVENT
}

\author{
ALAIN COCHARD and JAMES R. RICE \\ Division of Engineering and Applied Sciences and Department of Earth and Planetary Sciences, \\ Harvard University, Cambridge, MA 02138, U.S.A.
}

(Received 3 June 1996)

\begin{abstract}
Perrin et al. (1995) and Geubelle and Rice (1995) have introduced a spectral method for numerical solution of two- and three-dimensional elastodynamic fracture problems. The method applies for ruptures confined to a plane separating homogeneous elastic half spaces. In this method, the physical variables, such as the traction components of stress and displacement discontinuity on the rupture plane, are represented as Fourier series in space with time-dependent coefficients. An analytical solution is found for each Fourier mode, in that each Fourier coefficient for stress is expressed by the time convolution of the corresponding coefficient for displacement with a convolution kernel specific to the rupture mode. Once the 2D formulation of the method is known, the method is readily generalizable to 3D problems in that it involves only linear combinations of the convolution kernels found for each rupture mode in $2 \mathrm{D}$. This conceptual simplicity has, however, a major drawback : due to the Fourier series representations of the physical variables, the problem solved is in fact an infinite and periodic replication of rupture events on the fracture plane. So, in order to study the evolution of a single rupture, one has to use a spatial period large enough in order that the waves coming from the replication cracks do not enter the zone of interest during the time duration studied, or provide negligible stress alteration when they do arrive. We show here how to rigorously offset this defect while retaining the modal independence. Once expressed in the spatial domain, the method amounts to truncating in space the space-time convolution kernels, in a manner that provides an exact evaluation for all positions within the rupture domain (where the constitutive law between stress and displacement discontinuity is to be imposed), but not outside. In order for the method to be identical in structure to the method of Perrin et al. (1995) and Geubelle and Rice (1995), the period of the Fourier series is requested to be only twice as large as the rupture domain of interest. The only difference, then, to the original spectral method is that the convolution kernels in the Fourier domain require more elaborate calculations to be established, but this has to be done only once to allow simulations on a given domain. (c) 1997 Elsevier Science Ltd
\end{abstract}

Keywords : $\Lambda$. dynamic fracture, B. crack mechanics, C. fault dynamics, C. numerical algorithms.

\section{INTRODUCTION}

Perrin et al. (1995) and Geubelle and Rice (1995) have introduced a spectral method for numerical solution and two- and three-dimensional elastodynamic fracture problems. The method applies for ruptures confined to a plane separating homogeneous elastic half spaces. In this method, the physical variables, such as the traction components of stress and displacement discontinuity on the rupture plane, are represented as Fourier series in space with time-dependent coefficients. An analytical solution is 
found for each Fourier mode, in that each Fourier coefficient for stress is expressed, via a convolution integral, in terms of the corresponding coefficient for displacement. This modal independence allows for an efficient numerical method. It is highly suitable for implementation on parallel computers since the most time-consuming part of the calculation, namely evaluation of the convolution integrals, can be carried out in parallel without communication between processors.

This conceptual simplicity has, however a major drawback: due to the Fourier series representations of the physical variables, the problem solved is in fact an infinite and periodic replication of rupture events on the fracture plane. So, in order to study the evolution of a single rupture, one has to use a spatial period large enough in order that the waves coming from the replication cracks do not enter the zone of interest during the time duration studied, or provide negligible stress alteration when they do arrive. We show here how to offset this defect, dealing with just a single rupture event and yet retaining the modal independence which is critical to efficient calculations. The resulting spectral method without spatial replications requires more elaborate calculations to establish the convolution kernels, but is otherwise identical in structure to the method of Perrin et al. (1995) and Geubelle and Rice (1995). We describe the methodology in general, in 2D and 3D, and give full details of the analysis here for the 2D anti-plane strain (mode III) formulation.

Another spectral method has also been developed by Bouchon and Aki (1977) for kinematic representation of spatially extended sources, with $\exp (i \omega t)$ time dependence, in a vertically layered medium, representing the wave field by a Fourier series expansion in horizontal spatial coordinates. It has recently been applied to fracture problems (Bouchon and Streiff, 1997). Although different from Perrin et al. (1995) and Geubelle and Rice's (1995) method, it also presents the replication drawback mentioned above.

\section{THE SPECTR $\Lambda$ L METHOD FOR TWO-DIMENSIONAL PROBLEMS}

Consider a two-dimensional displacement field, dependent on $x, y$, and $t$, and associated with rupture along the plane $y=0 \mathrm{in}$ an infinite homogeneous space. Let $\delta(x, t)=u\left(x, y=0^{+}, t\right)-u\left(x, y=0^{-}, t\right)$ and $\tau(x, t)$ denote the displacement discontinuity and the stress on the rupture plane, respectively. This $\delta$ and $\tau$ may refer to opening displacement and tensile stress (mode I), or to in-plane (mode II) an antiplane (mode III) slip and associated shear stress.

According to the equations of elastodynamics, the relation between stress and displacement discontinuity can be expressed by [e.g. Geubelle and Rice (1995)]

$$
\tau(x, t)=\tau^{0}(x, t)-\Lambda \delta_{, t}(x, t)+f(x, t)
$$

in which $\tau^{0}$ is the externally applied stress (i.e. that which would act if the rupture plane was constrained against displacement discontinuity), $\Lambda$ is the radiation damping term, equal to $\mu / 2 c_{\mathrm{s}}$ for modes II and III and $(\lambda+2 \mu) / 2 c_{\mathrm{d}}$ for mode I, where $\lambda$ and $\mu$ are the Lamé parameters and $c_{\mathrm{s}}$ and $c_{\mathrm{d}}$ are the shear and dilational wave speeds, 
respectively. The subscripted comma denotes differentiation, and $f(x, t)$ is a linear functional of previous displacement history.

Perrin et al. (1995) for mode III and Geubelle and Rice (1995) for the other modes show that if the displacement is expressed as $\delta(x, t)=D(k, t) \exp (\mathrm{i} k x)$, then this functional is $f(x, t)=F(k, t) \exp (\mathrm{i} k x)$ with

$$
F(k, t)=-\frac{\mu c_{\mathrm{s}}|k|^{2}}{2} \int_{-\infty}^{t} C\left(|k| c_{\mathrm{s}}(t-\theta)\right) D(k, \theta) \mathrm{d} \theta,
$$

where the expressions for the convolution kernels $C(T)$ for each mode are given in Appendix A. Thus, if we write the slip as a continuous superposition of Fourier modes

$$
\delta(x, t)=\frac{1}{2 \pi} \int_{-\infty}^{+\infty} D(k, t) e^{i k x} \mathrm{~d} k
$$

[by which we also define the Fourier transform $\tilde{\delta}(k, t)=D(k, t)]$, we have the following spectral formulation of the functional $f$ in equation (1):

$$
f(x, t)=\frac{1}{2 \pi} \int_{-\infty}^{+\infty} F(k, t) \mathrm{e}^{i k x} \mathrm{~d} x .
$$

For numerical tractability, $D(k, t)$ is concentrated at discrete wavenumbers $k_{n}=2 \pi n / \lambda$, where $n$ is integer, so as to represent $\delta(x, t)$ as Fourier series summation of modes consistent with a period of length $\lambda$ along the $x$ axis,

$$
\delta(x, t)=\sum_{n=-\infty}^{+\infty} D_{n}(t) \mathrm{e}^{2 i \pi n x / \lambda} .
$$

Then the functional $f(x, t)$ is given, for all $x$, as

$$
f(x, t)=\sum_{n=-\infty}^{+\infty} F_{n}(t) \mathrm{e}^{2 \mathrm{i} \pi n x / \lambda},
$$

where $F_{n}(t)$, derived from $F(k, t)$ with $k=2 \pi n / \lambda$, is :

$$
F_{n}(t)=-\frac{\mu c_{\mathrm{s}}}{2}\left(\frac{2 \pi n}{\lambda}\right)^{2} \int_{-\infty}^{t} C\left(\left|\frac{2 \pi n}{\lambda}\right| c_{\mathrm{s}}(t-\theta)\right) D_{n}(\theta) \mathrm{d} \theta .
$$

In using this as a numerical method for crack propagation, the summations in (5) and (6) are truncated at $-N / 2$ and $+N / 2$ (the larger the $N$-necessarily an even number-the more accurate the solution) and the following procedure is implemented: we suppose the displacement history is known up to the present time $t$. The fast Fourier transform (FFT) is used to obtain $D_{n}(t)$ from $\delta(x, t)$ at $N$ sample points $x=j \Delta x$, within a period, where $j=0,1, \ldots, N-1$ and $\Delta x=\lambda / N$ is the sample point spacing. For this purpose, the choice $N=2^{p}$ is made where $p$ is a positive integer. The same has been done after previous time steps on the calculation so that this extends the history of $D_{n}(\theta)$ to time $\theta=t$. The time convolution is then explicitly computed giving $F_{n}(t)$ and finally an inverse FFT gives the functional $f(j \Delta x, t)$. Once $f(x, t)$ is thus known, the velocity $\delta_{. t}(x, t)$ at that time can be computed by 
simultaneously solving (1) together with some constitutive relation between $\tau$ and $\delta$, and possibly $\delta_{, t}$. For example, Geubelle and Rice (1995) modeled mode I cracking with a simple cohesive model with linear displacement-weakening from peak to zero strength. More complex constitutive laws have been used to characterize shear rupture surfaces, such as rate- and state-dependent friction laws (Perrin et al., 1995) which relate the strength to both the slip velocity and a state variable whose value depends on previous slip history. Knowing the slip velocity, $\delta(x, t+\Delta t)$ at the next time step can be determined, hence $f(x, t+\Delta t)$, and so on.

The Fourier series representation of $\delta(x, t)$ [equation (5)] shows that the slip history is spatially periodic with replication period $\lambda$, requiring, as already said, that $\lambda$ be chosen much wider than the crack length actually studied in order to avoid or acceptably reduce interaction from replication cracks.

\section{THE SPECTRAL METHOD WITHOUT REPLICATION, TWO- DIMENSIONAL PROBLEMS}

Equation (2) can be rewritten as

$$
\tilde{f}(k, t)=-\frac{\mu c_{\mathrm{s}}}{2} k^{2} \tilde{g}(k, t)
$$

where

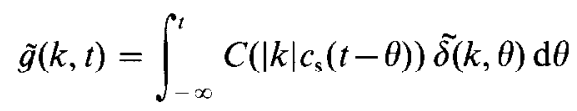

in which

$$
\tilde{\delta}(k, \theta)=\int_{-\infty}^{+\infty} \delta(\xi, \theta) \mathrm{e}^{-i k \xi} \mathrm{d} \xi .
$$

If we want to study a single rupture which is always confined within a spatial region, say, between $-L / 2$ and $+L / 2$ (see Fig. 1), this means that $\delta$ will be non-zero only in this domain. This is equivalent to substituting $[-L / 2,+L / 2]$ for the domain of integration in the previous equation. We want to use the same methodology as in the previous section, i.e. to write $\delta$ as a Fourier series and to obtain $f$ as a similar series.

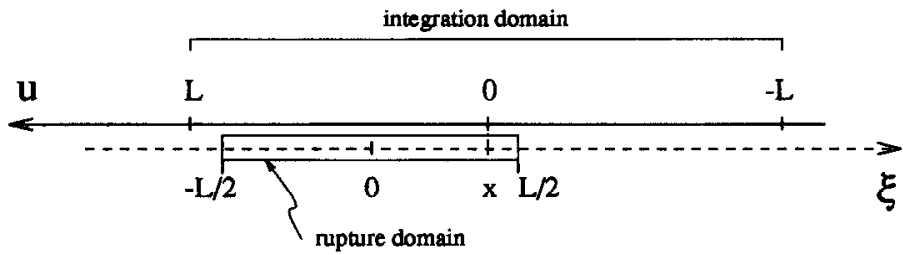

Fig. 1. Rupture domain of length $L$ along the $\xi$ axis and variable (with $x$ ) actual domain of integration, always of length $2 L$ centered on position $x$ as marked above, for the computation of $\tilde{\delta}(k, \theta)$ in (9) or for that of $K(n, t)$ in (35) for the implementation of the spectral method without replication in two dimensions. 
The key point in allowing this, and achieving the desired modal independence, is then to notice that

$$
\int_{-L / 2}^{+L / 2} \varphi(\xi) \mathrm{d} \xi=\int_{x-L}^{x+L} \varphi(\xi) \mathrm{d} \xi \text { for } x \in\left[-\frac{L}{2},+\frac{L}{2}\right]
$$

for any function $\varphi(\xi)$ that is zero on $[-3 L / 2,-L / 2]$ and $[+L / 2,+3 L / 2]$. This property is achieved if we write $\delta$ as a Fourier series with period $2 L$, to be used exclusively on the domain $[-3 L / 2,+3 L / 2]$,

$$
\delta(\xi, \theta)=\sum_{n=-\infty}^{+\infty} D_{n}(\theta) \mathrm{e}^{2 i \pi n \xi / 2 L} .
$$

This series is defined by the values of $\delta(\xi, \theta)$ for $\xi \in]-L,+L[$, and these values vanish on the part $[-L,-L / 2]$ and $[+L / 2,+L]$ of that domain. Hence, since the series sums to a periodic function, it sums to zero on $[-3 L / 2,-L / 2]$ and $[+L / 2,+3 L / 2]$ as needed for (10).

Thus we may write (9) as

$$
\begin{aligned}
\tilde{\delta}(k, \theta) & =\tilde{\delta}(k, \theta ; x)=\int_{x-L}^{x+L}\left(\sum_{n=-\infty}^{+\infty} D_{n}(\theta) \mathrm{e}^{2 \mathrm{i} \pi n \xi / 2 L}\right) \mathrm{e}^{-\mathrm{i} k \xi} \mathrm{d} \xi \\
& =\left(\sum_{n=-\infty}^{+\infty} D_{n}(\theta) \frac{2 \sin (k L-n \pi)}{k-n \pi / L} \mathrm{e}^{2 \mathrm{i} \pi n x / 2 L}\right) \mathrm{e}^{-\mathrm{i} k x},
\end{aligned}
$$

which is actually independent of $x$ for $x \in[-L / 2,+L / 2]$. The advantage of this otherwise unusual step is that it will lead us to a Fourier series in the same form as (11) for $f(x, t)$. That series will be valid only for $x \in[-L / 2,+L / 2]$, which is where we need $f(x, t)$ in the numerical algorithm, and has each Fourier coefficient for $f$ dependent only on the history of the corresponding coefficient for $\delta$, such now being achieved without spatial replications of the rupture event. We give another, fully equivalent, route to the same objective in Section 4 below.

Thus, from (8) we get

$$
\tilde{g}(k, t)=\tilde{g}(k, t ; x)=\sum_{n=-\infty}^{+\infty} \int_{-\infty}^{t} C\left(|k| c_{\mathrm{s}}(t-\theta)\right) D_{n}(\theta) \frac{2 \sin (k L-n \pi)}{k-n \pi / L} \mathrm{~d} \theta \mathrm{e}^{-\mathrm{i} k x} \mathrm{e}^{2 \mathrm{i} \pi n x / 2 L},
$$

which is likewise actually independent of $x$, for $x$ on $[-L / 2,+L / 2]$. Thus, if we need $g(x, t)$ only for $x \in[-L / 2,+L / 2]$, we can write

$$
g(x, t)=\frac{1}{2 \pi} \int_{-\infty}^{+\infty} \tilde{g}(k, t ; x) \mathrm{e}^{\mathrm{i} k x} \mathrm{~d} k, \quad x \in[-L / 2,+L / 2] .
$$

Noting now that $\tilde{f}(k, t)=-\left(\mu c_{\mathrm{s}} / 2\right) k^{2} \tilde{g}(k, t)$ from (8) is equivalent to $f(x, t)=$ $\left(\mu c_{\mathrm{s}} / 2\right) \partial^{2} g(x, t) / \partial x^{2}$, we have the Fourier series representation 


$$
f(x, t)=\sum_{n=-\infty}^{+\infty} F_{n}^{*}(t) \mathrm{e}^{2 \mathrm{i} \pi n x / 2 L}, \quad x \in[-L / 2,+L / 2],
$$

where

$$
F_{n}^{*}(t)=-\frac{\mu c_{\mathrm{s}}}{2}\left(\frac{\pi n}{L}\right)^{2} \int_{-\infty}^{t} K(n, t-\theta) D_{n}(\theta) \mathrm{d} \theta
$$

with

$$
K(n, t)=\frac{(-1)^{n}}{\pi} \int_{-\infty}^{+\infty} C\left(|k| c_{\mathrm{s}} t\right) \frac{\sin (k L)}{k-n \pi / L} \mathrm{~d} k
$$

Thus, unlike in the formulation with replications, in which the expressions for the convolution kernels for each spectral mode are given by a single expression, as in equation (7), with the mode number $n$ appearing only as a scaling factor for the time axis, for the formulation without replication, there is a separate expression for the convolution kernel for each spectral mode. This is also what happens in the viscoelastic case, even for the formulation with replication, as shown by Geubelle et al. (1997).

In the numerical implementation, the sums in (11) and (15) extend not from $-\infty$ to $+\infty$, but from $-N / 2$ to $+N / 2$. The procedure is identical to that described after equation (7).

The following section outlines the practical numerical evaluation of $K(n, t)$ for mode III. A similar procedure may also be followed for the other rupture modes, as will be seen in Section 4 below.

\subsection{Kernel equation for the case of mode III rupture}

For mode III we use $C(T)=C_{\mathrm{III}}(T)$, Appendix A. Perrin et al. (1995) determined the expression of $C_{\mathrm{III}}(T)=J_{1}(T) / T$ from an integral representation which is

$$
C_{\mathrm{III}}\left(|k| c_{\mathrm{s}} t\right)=\frac{1}{2 \pi} \int_{0}^{2 \pi} \cos ^{2} \psi \mathrm{e}^{\mathrm{i}|\hbar| \bar{c}_{\mathrm{s}} \sin \psi} \mathrm{d} \psi
$$

Inserting this expression into representation (17) of $K(n, t)$, then writing $\sin (k L)$ as $[\exp (\mathrm{i} k L)-\exp (-\mathrm{i} k L)] / 2 \mathrm{i}$ and further noticing that

$$
\mathrm{e}^{\mathrm{i} k\left( \pm L+c_{\mathrm{s}} t \sin \psi\right)}=\mathrm{e}^{\mathrm{i}(k-n \pi / L)\left( \pm L+c_{\mathrm{s}} t \sin \psi\right)} \mathrm{e}^{\mathrm{i} n \pi\left[ \pm 1+\left(c_{\mathrm{s}} t / L\right) \sin \psi\right]}
$$

we can perform the integration on $k$ and we get

$$
K(n, t)=\frac{1}{4 \pi} \int_{0}^{2 \pi} \cos ^{2} \psi \mathrm{e}^{\mathrm{i}\left(m a c_{\mathrm{s}} / L\right) \sin \psi}\left[\operatorname{sign}\left(L+c_{\mathrm{s}} t \sin \psi\right)-\operatorname{sign}\left(-L+c_{\mathrm{s}} t \sin \psi\right)\right] \mathrm{d} \psi .
$$

We can see that the real part of the integrand is even with respect to $\psi=\pi$ whereas the imaginary part is odd with respect to $\psi=\pi$ (so its contribution is zero). Also on $[0, \pi]$, the first sign is always equal to 1 and, noting that the real part is symmetric relative to $\pi / 2$, we have 


$$
K(n, t)=\frac{1}{\pi} \int_{0}^{\pi / 2} \cos ^{2} \psi \cos \left(\frac{n \pi c_{\mathrm{s}} t}{L} \sin \psi\right)\left[1-\operatorname{sign}\left(-L+c_{\mathrm{s}} t \sin \psi\right)\right] \mathrm{d} \psi .
$$

If $c_{\mathrm{s}} t / L<1$, the present method should agree with the original spectral method (with its replications) summarized in Section 2 with $\lambda=2 L$, since for such $t$ the waves coming from the two nearest replication cracks have not yet reached the domain of interest. Observing that $\operatorname{sign}\left(-L+c_{s} t \sin \psi\right)=-1$ for such times, we have

$$
K(n, t)=\frac{1}{2 \pi} \int_{0}^{2 \pi} \cos ^{2} \psi \cos \left(\frac{n \pi c_{\mathrm{s}} t}{L} \sin \psi\right) \mathrm{d} \psi,
$$

which agrees with $C_{\mathrm{III}}\left(|k| c_{\mathrm{s}} t\right)$ of the original spectral method, with $k=n \pi / L$, as expected, and can likewise be computed with polynomial approximations. By contrast, if $c_{\mathrm{s}} t / L>1$, we have to make the sign explicit to get

$$
K(n, t)=\frac{2}{\pi} \int_{0}^{\psi_{1}} \cos ^{2} \psi \cos \left(\frac{n \pi c_{s} t}{L} \sin \psi\right) \mathrm{d} \psi,
$$

where $\psi_{1}=\arcsin \left(L / c_{\mathrm{s}} t\right)$. This can be rewritten as

$$
K(n, t)=\frac{1}{2 \pi}\left\{\int_{0}^{2 \pi}-4 \int_{\psi_{1}}^{\pi / 2}\right\} \cos ^{2} \psi \cos \left(\frac{n \pi c_{\mathrm{s}} t}{L} \sin \psi\right) \mathrm{d} \psi,
$$

where (18) is used for the first integral. The integrals with a limit $\psi_{1}$ have to be computed numerically. When $c_{\mathrm{s}} t / L=1, \psi_{1}=\pi / 2$ whereas when $t \rightarrow+\infty, \psi_{1} \rightarrow 0$ so formulation (24) is numerically advantageous for small $t$ whereas formulation (23) is better for large $t$. We used a classical integration routine with Simpson's rule. Figure 2 shows the variation of the new, non-replicating kernel $K(n, t)$ with time for some

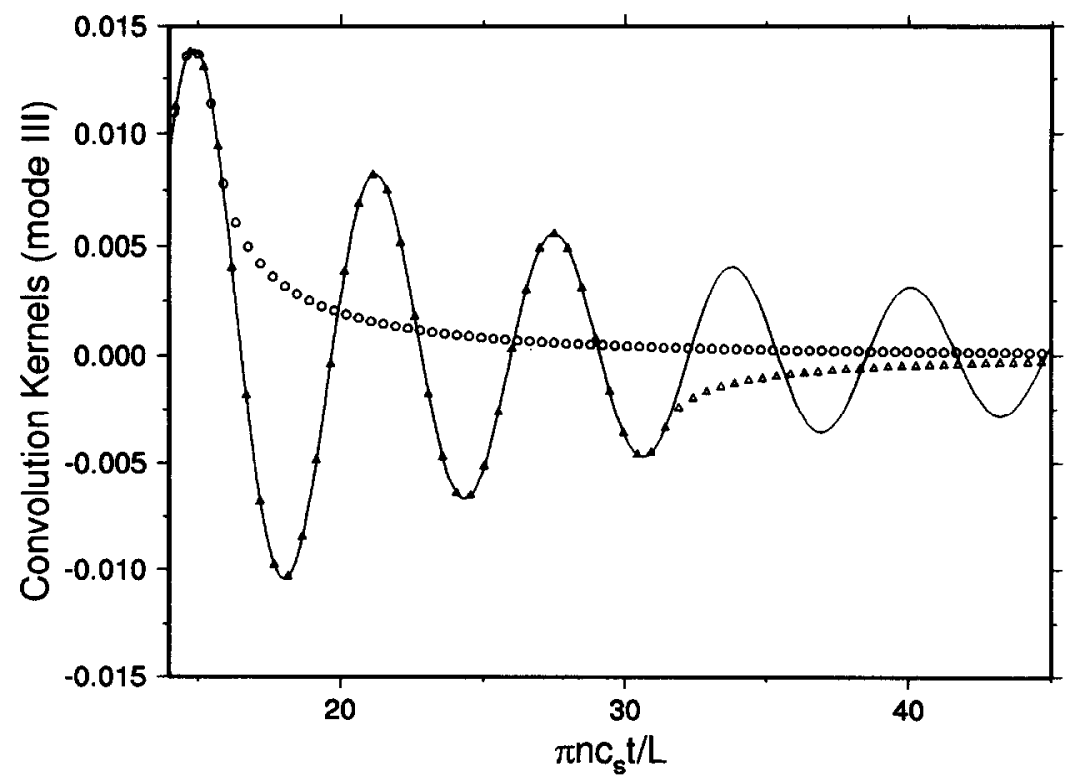

Fig. 2. Convolution kernels for mode III, for the formulation with replication and any mode (full line) and for the formulation without replication for mode $n=5$ (circles) and mode $n=10$ (triangles). 
mode numbers $n$, together with its counterpart, $J_{1}\left(\pi n c_{s} t / L\right) /\left(\pi n c_{s} t / L\right)$, in the previous formulation with periodic replication. Unlike the replication kernel, the new kernel does not oscillate any more when $c_{\mathrm{s}} t / L>1$ and tends to zero with negative values for even modes (like for $n=10$ in Fig. 2) and positive values for odd modes $(n=5)$. The new kernel for $n=1$ does not oscillate at all, but decays monotonically.

\subsection{Numerical illustration for mode III}

To illustrate the above methodology, we study the problem of the dynamic loading of a non-propagating finite crack of length $L$ under instantaneous spatially uniform stcp loading at time 0 , i.e. $\tau^{0}(x, t)=\tau_{0} U(t)$, where $U(t)$ is the unit step function. The friction is assumed to be zero but when the velocity of a particular point becomes negative this point is supposed to "freeze", i.e. no backward motion is allowed so that we can observe the evolution of the stress.

In the numerical simulations to be presented, we have chosen the parameter $\beta=c_{\mathrm{s}} \Delta t / \Delta x=0.5$ where $\Delta t$ is the time step. Also, it has been shown (Morrissey and Geubelle, 1997) that the accuracy of the solution for mode III problems can be improved by introducing an "artificial" delay $\varepsilon_{t} \Delta t$, a fraction of $\Delta t$, in the discretized version of the time convolution which involves the summation $\sum_{j=0}^{\mathrm{i} t} X K\left[n,\left(\mathrm{i} t-j+\varepsilon_{t}\right) \Delta t\right] D_{n}(j \Delta t) . X K(n, t)$ is the result of an operator on $K(n, t)$ which can either simply be identity (i.e. $X K(n, t)=K(n, t))$ or, as in the present study, a more elaborate operator leading to the result that $X K(n, t)$ is a "pre-integrated" value of $K(n, t)$ between $t-\Delta t$ and $t$, using a trapezoidal-rule integration (Geubelle and Rice, 1995). The optimal value of the delay for mode III is $\varepsilon_{t}=0.5$, which is the value that has been used in the simulations presented here. $\dagger$ Finally, the time marching scheme used in the present study is fully explicit, i.e. $D_{n}(t+\Delta t)=D_{n}(t)+\Delta t \dot{D}_{n}(t)$.

Figure 3 illustrates both the effectiveness of the method and the potential computational gain. The top panel represents the evolution of the stress (normalized by the magnitude of the initial step loading) versus normalized time at the center of the crack $(x=0)$. One of the three curves is the result of a simulation using the method with spatial replication and a replication period $\lambda=2 L$. Another such simulation with $\lambda=8 L$ gives the second curve whereas the third curve has been obtained using the method described in the previous section, i.e. without replication in space and with $\lambda=2 L$. For the study of a domain in which rupture occurs over length $L$, this is the minimum allowed value if the method is used. The bottom panel is a schematic space-time illustration of the problem, showing the replication cracks and the time at which they interact with the original crack (the problem is of course symmetric with respect to $x=0$ but only the negative part has been displayed for conciseness). The evolutions computed without replication on the one hand and with replication and $\lambda=8 L$ on the other hand, coincide up to the time $\left(c_{s} t / L=7.5\right)$ where the effect of the first replication crack is felt. This shows that our method gives good results (at least up to this time).

Suppose now that we want to compute the evolution of the whole crack (not only for the central point) up to time $7 L / c_{\mathrm{s}}$, without having any effects from the replication

$\dagger$ This delay seems to have an effect very similar to that introduced in choosing time collocation points in Cochard and Madariaga (1994). 


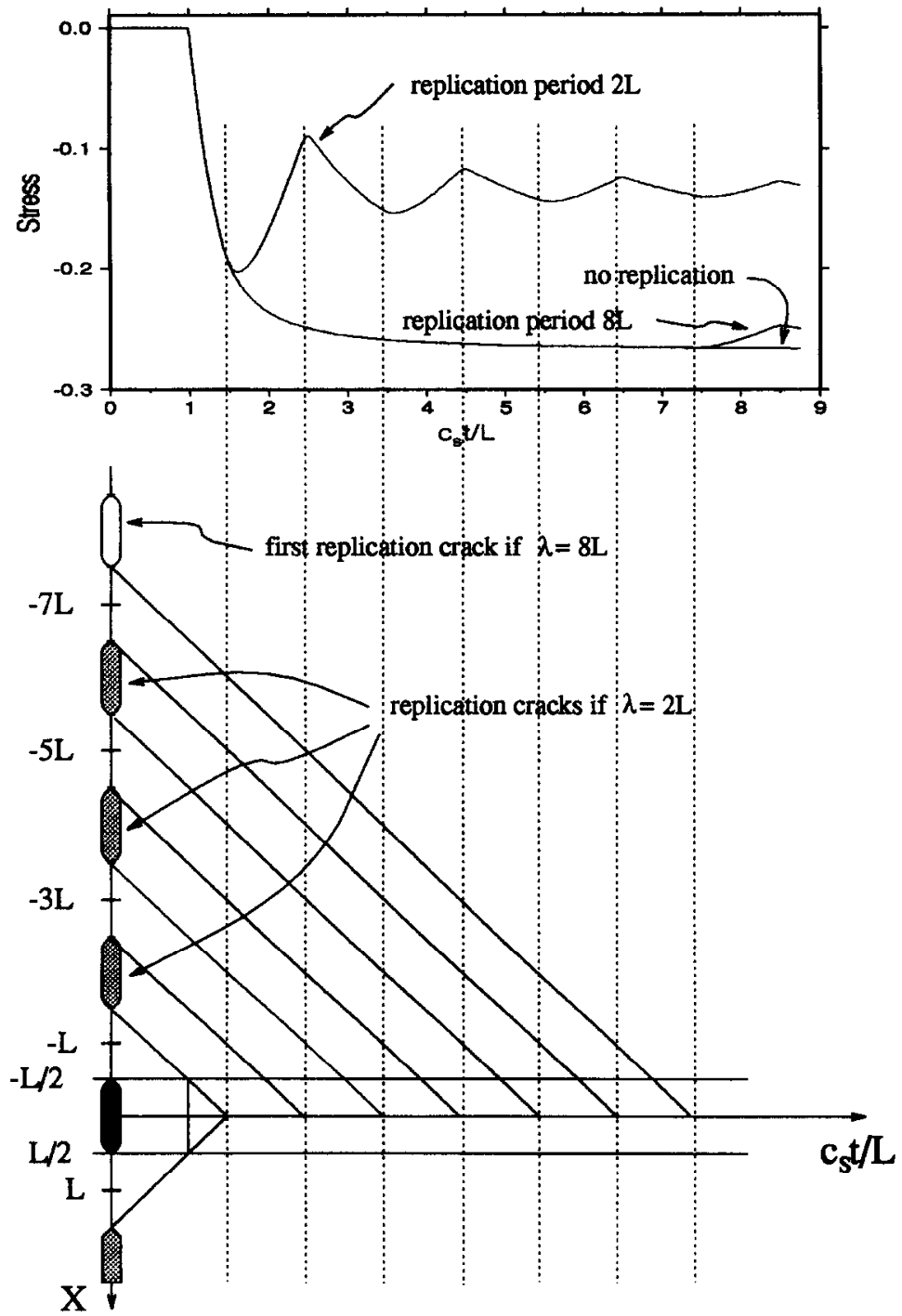

Fig. 3. Top: evolution of stress versus normalized time at the center of a non-propagating crack submitted to an instantaneous spatially uniform step loading at time 0 computed with the spectral method with two different replication periods and without replication. Bottom: schematic space-time illustration of the problem, showing the replication cracks and the time at which they interact with the original crack.

ruptures. Then, if we use the method with spatial replication, we have to use a replication period $\lambda=8 L$. The computation thus involves four times as many operations as with the new method and, besides, we get rid of the unwanted replications. For the analog problem in $3 \mathrm{D}$, the gain would be $4 \times 4=16$. We also see that the new formulation becomes advantageous as soon as we want to compute the whole crack evolution for $c_{s} t / L>1$, i.e. as soon as the waves coming from the nearest replication crack arrive at $x= \pm L / 2$. For smaller times, we could use a replication period smaticr than $2 L$ with the method with replication and save time. 
The numerical integration required for computing the convolution kernel would not make the method advantageous for only one simulation. However, if the number of spectral modes used and time discretization are identical from one simulation to the other one, we need not compute the kernel again (one could also use a kernel obtained with a different time discretization and interpolate to the time values actually needed).

\section{ALTERNATIVE TWO-DIMENSIONAL DERIVATION STARTING FROM THE SPATIO-TEMPORAL REPRESENTATION}

\subsection{Spatio-temporal convolution}

Let us consider the general problem of representing the relation between the displacement discontinuity and stress distributions. We begin with (8) and take the Laplace transform, defined by

$$
\hat{\tilde{f}}(k, p)=\int_{0}^{+\infty} \mathrm{e}^{-p} \tilde{f}(k, t) \mathrm{d} t
$$

Then (8) becomes

$$
\hat{\tilde{f}}(k, p)=-\frac{\mu c_{\mathrm{s}}}{2} k^{2} \hat{\tilde{g}}(k, p), \quad \hat{\tilde{g}}(k, p)=\frac{1}{|k| c_{\mathrm{s}}} \mathscr{M}\left(\frac{p}{|k| c_{\mathrm{s}}}\right) \hat{\tilde{\delta}}(k, p)
$$

Here it has been recalled that a convolution on time becomes a product of transforms and the functions $\mathscr{M}(s)$, different for each of the three modes, are defined by

$$
\mathscr{M}(s)=\int_{0}^{+\infty} \mathrm{e}^{-s T} C(T) \mathrm{d} T .
$$

In fact, on their way to deriving the expressions for $C(T)$ summarized in Appendix A, Geubelle and Rice (1995) obtained in their equations (24) the relations between $\hat{f}$ and $\hat{\tilde{\delta}}$ for the three modes. From those expressions we may read off the expressions corresponding to $\mathscr{M}(s)$ [except for a misprint: the term in curly brackets in the last of their equations (24) should be $\alpha_{s}-p /|q| c_{s}$ ]. Those functions, all analytic in $\operatorname{Re}(s)>0$, and branch cut so that $\mathscr{M}(s) \rightarrow 0$ as $s \rightarrow+\infty$, are listed in Appendix B. Now, let $G(x, t)$ be the function whose Fourier-Laplace transform is

$$
\hat{\tilde{G}}(k, p)=\frac{1}{|k| c_{\mathrm{s}}} \mathscr{M}\left(\frac{p}{|k| c_{\mathrm{s}}}\right) .
$$

The significance of this function (actually, one for each mode) is that $g(x, t)$ has the space-time convolution form

$$
g(x, t)=\int_{-\infty}^{t} \int_{-\infty}^{+\infty} G(x-\xi, t-\theta) \delta(\xi, \theta) \mathrm{d} \xi \mathrm{d} \theta .
$$

Since $\mathscr{M}(s)$ is the Laplace transform, with factor $\exp (-s T)$, of $C(T)$, it is clear that 


$$
\widetilde{G}(k, t)=C\left(|k| c_{s} t\right)
$$

is the function whose Laplace transform, with factor $\exp (-p t)$, gives $\hat{\widetilde{G}}(k, p)$ above. Thus

$$
\begin{aligned}
G(x, t) & =\frac{1}{2 \pi} \int_{-\infty}^{+\infty} \mathrm{e}^{\mathrm{i} k x} C\left(|k| c_{\mathrm{s}} t\right) \mathrm{d} k \\
& =\frac{1}{2 \pi} \int_{0}^{+\infty}\left(\mathrm{e}^{\mathrm{i} k x}+\mathrm{e}^{-\mathrm{i} k x}\right) C\left(k c_{\mathrm{s}} t\right) \mathrm{d} k \\
& =\frac{1}{2 \pi c_{\mathrm{s}} t} \int_{0}^{+\infty}\left[\mathrm{e}^{\mathrm{i}\left(x / c_{\mathrm{s}} t\right) T}+\mathrm{e}^{-\mathrm{i}\left(x / c_{\mathrm{s}} t\right) T}\right] C(T) \mathrm{d} T \\
& =\frac{1}{2 \pi c_{\mathrm{s}} t}\left[\mathscr{M}\left(-\mathrm{i} x / c_{\mathrm{s}} t+0\right)+\mathscr{M}\left(\mathrm{i} x / c_{\mathrm{s}} t+0\right)\right] \\
& =\frac{1}{\pi c_{\mathrm{s}} t} \operatorname{Re}\left[\mathscr{M}\left(\mathrm{i} x / c_{\mathrm{s}} t+0\right)\right],
\end{aligned}
$$

with the last form arising since, in our situation, $C(T)$ is real for real $T$ and hence $\mathscr{M}(s)$ has complex conjugate $\overline{\mathscr{M}(s)}=\mathscr{M}(\bar{s})$. Willis (1973), and Willis and Movchan (1995) in their Appendix B, present a related way of inverting transforms like those for $\hat{G}(k, p)$ above, and our result is consistent with theirs.

Hence, defining $M\left(x / c_{\mathrm{s}} t\right)=\operatorname{Re}\left[\mathscr{M}\left(\mathrm{i} x / c_{\mathrm{s}} t+0\right)\right]$, we report expressions for the functions $M(u)$ for the three modes in Appendix B. Finally, as we know, from expression (8) for $\tilde{g}$, that $f(x, t)=\left(\mu c_{\mathrm{s}} / 2\right) \partial^{2} g(x, t) / \partial x^{2}$,

$$
f(x, t)=\frac{\mu c_{\mathrm{s}}}{2 \pi} \frac{\partial^{2}}{\partial x^{2}}\left(\int_{-\infty}^{t} \int_{-\infty}^{+\infty} M\left(\frac{x \quad \xi}{c_{\mathrm{s}}(t-\theta)}\right) \frac{\delta(\xi, \theta)}{c_{\mathrm{s}}(t-\theta)} \mathrm{d} \xi \mathrm{d} \theta\right) .
$$

Equivalently, integrating twice by parts with respect to $\xi$ and assuming that $\delta(\xi, \theta)$ and $\partial \delta(\xi, \theta) / \partial \xi$ vanish when $\xi \rightarrow \pm \infty$, we have

$$
f(x, t)=\frac{\mu c_{\mathrm{s}}}{2 \pi} \int_{-\infty}^{t} \int_{-\infty}^{+\infty} M\left(\frac{x}{c_{\mathrm{s}}(t-\theta)}\right) \frac{\delta_{. \xi \xi}(\xi, \theta)}{c_{\mathrm{s}}(t-\theta)} \mathrm{d} \xi \mathrm{d} \theta .
$$

This may be shown to be consistent with the form used by, e.g., Cochard and Madariaga (1994) and Kostrov (1966) in mode III.

\subsection{Alternative derivation of spectral method without replications}

Now let us formulate the spectral method without replication for a rupture which, again, has non-zero $\delta$ only within the domain $]-L / 2,+L / 2[$ (see Fig. 1). Thus, in (32) or (33), we want to integrate over $\xi$ for $\xi \in[-L / 2,+L / 2]$ instead of $\xi \in]-\infty,+\infty$ [. Then, similarly to what was done in Section 3 , changing the domain of integration to $[x-L, x+L]$, inserting the Fourier series representation (11) of $\delta(\xi, \theta)$ with period $2 L$ and finally making the change of variables $u=x-\xi$, we get 


$$
f(x, t)=-\frac{\mu c_{\mathrm{s}}}{2 \pi} \sum_{n=-\infty}^{+\infty}\left(\frac{\pi n}{L}\right)^{2} \int_{-\infty}^{t} D_{n}(\theta) \int_{-L}^{+L} M\left(\frac{u}{c_{\mathrm{s}}(t-\theta)}\right) \frac{\mathrm{e}^{-2 i \pi n u / 2 L}}{c_{\mathrm{s}}(t-\theta)} \mathrm{d} u \mathrm{~d} \theta \mathrm{e}^{2 \mathrm{i} \pi n x / 2 L}
$$

for $x \in[-L / 2,+L / 2]$. This is a Fourier series in the form of $(15,16)$ where now the innermost integral provides the expression for $K(n, t)$ as

$$
K(n, t)=\int_{-L}^{+L} \frac{M\left(u / c_{\mathrm{s}} t\right)}{\pi c_{\mathrm{s}} t} \mathrm{e}^{-2 \mathrm{i} \pi n u / 2 L} \mathrm{~d} u,
$$

or, taking into account the evenness of $M$ and making the change of variables $p=u / c_{\mathrm{s}} t$ :

$$
K(n, t)=\frac{2}{\pi} \int_{0}^{L / c_{\mathrm{s}} t} M(p) \cos \left(\pi n p c_{\mathrm{s}} t / L\right) \mathrm{d} p .
$$

These expressions for $K(n, t)$ are, of course, equivalent to its expression (17) found from a different approach. To see this, one has to write for $\left(1 / \pi c_{s} t\right) M\left(x / c_{s} t\right)=G(x, t)$ the expression in the first equality of (31), then set $x=u$ and insert into equation (35), exchange integral signs and integrate over $u$, which provides the result.

If we had implemented the (original) spectral method with replication, i.e. keeping ]$-\infty,+\infty[$ as the integration domain instead of changing it to $[-L / 2,+L / 2]$ in expression (33) for $f(x, t)$, the upper integration limit in expression (36) for $K(n, t)$ would be $+\infty$. On the other hand, $M(u)=0$ for $|u|>u_{\max }\left(u_{\max }=1\right.$ or $c_{\mathrm{d}} / c_{\mathrm{s}}$ depending on mode, see Appendix B), so that, in this case, the upper limit of integration in (36) could be changed to $+\infty$. So, for $L / c_{\mathrm{s}} t<u_{\max }$, i.e. a short cnough time $t$, the formulations of the spectral method with and without replication are rigorously equivalent.

Let us also note that making the change of variables $p=\sin \psi$ in (36) when evaluated for the mode III case immediately provides expression (24). This approach for finding the convolution kernels thus seems to be a very general route to provide an efficient way of computing them numerically.

Finally, it can be seen from (35) that $K(n, t)$ is related to the Fourier coefficient of $M$ :

$$
\frac{1}{\pi c_{\mathrm{s}} t} M\left(\frac{u}{c_{\mathrm{s}} t}\right)=\frac{1}{2 L_{n}} \sum_{n=-\infty}^{+\infty} K(n, t) \mathrm{e}^{2 i \pi n u / 2 L} .
$$

We can thus approximate $K(n, t)$ with the discrete Fourier transform (using FFT), so that if the summation extends from $-N / 2$ to $+N / 2$ in (11) and (15), the $N$ kernels $K(n, t)$ are given in terms of linear combinations of $2 L M\left(x / c_{\mathrm{s}} t\right) / \pi c_{\mathrm{s}} t$ at the $N$ FFT sample points along the $x$ axis. In the next section we present an illustration of the use of this approximation for mode III.

\subsection{Approximation of the convolution kernel by discrete Fourier series (mode III case)}

As suggested above, we can approximate $K(n, t)$ by taking the fast Fourier transform of $\left(2 L / \pi c_{\mathrm{s}} t\right) M\left(x / c_{\mathrm{s}} t\right)=2 L \sqrt{1-\left(x / c_{\mathrm{s}} t\right)^{2}} U\left(c_{\mathrm{s}} t-|x|\right)$ for mode III, $U$ being the Heaviside function (see Appendix B). 


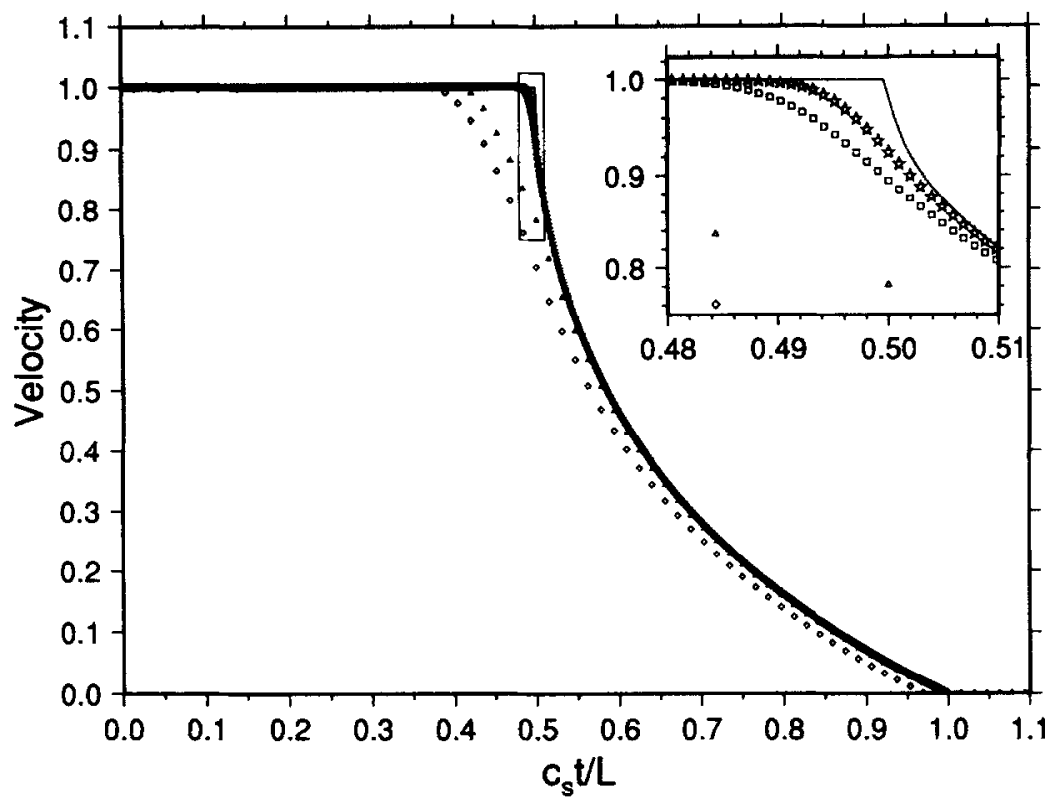

Fig. 4. Evolution of slip velocity versus normalized time for the same problem as in Fig. 3 and also at the center of the crack. Comparison to analytical solution (solid line) of numerical solutions computed for two different values of the number $N$ of summation terms involved in the Fourier series, with two different versions of the spectral method in which the convolution kernels are rigorously evaluated $(N=64$, triangles; $N=1024$, stars) or only approximately $(N=64$, diamonds; $N=1024$, squares). The advantage of the "approximate" method is that spatial replication is avoided without requiring sophisticated evaluation of the convolution kernels (see text). Inset : blow-up of the rectangular area.

We use this method for the same problem as studied in Section 3.2 and present the results (Fig. 4) for the evolution of the velocity of the central point of the crack for $c_{\mathrm{s}} t / L<1$. For such small times, the solutions can be compared with the analytical expressions obtained by Burridge (1969). Velocity is normalized by $2 c_{\mathrm{s}} / \mu$ times the magnitude of the initial step loading. The crack is discretized by 32 or 512 elements (number of elements $=N / 2$ ) in the two cases presented and the numerical solution is compared to the numerical solution obtained without any approximation, other than numerical integration, in evaluation of the convolution kernels (i.e. as derived in Section 3, or equivalently 4.2 and illustrated in Section 3.2) and to the analytical solution.

If the delay $\varepsilon_{t}=0$ (see Section 3.2), the numerical solution is completely unstable for either discretization, unlike the solution obtained without the approximation of this section, which is stable for every value of the delay between 0 and 1 .

For the optimal value of $\varepsilon_{t}=0.5$, when the fault is discretized by 32 elements, the numerical solution obtained using the approximate kernel evaluation (diamonds) is much worse than the solution obtained without using the approximation (triangles). The solution is of course better when 512 elements are used, but the solution with the approximate kernels (squares) is still worse than with exact kernels (stars) especially in the vicinity of $c_{\mathrm{s}} t / L=0.5$, which is the time at which the waves coming from the edges of the crack arrive at its center. So the only advantage of using this approxi- 
mation of the kernel by Fourier series is that the kernel evaluation is much fasterto the detriment of accuracy-but could be preferred, for example, if we had to perform only a few simulations for some unusual parameter range, such that it would not be worth computing the exact values of the kernels.

\section{2D STATIC CRACK ANALYSIS : SPECTRAL METHOD WITHOUT REPLICATION}

The well-known relations between stress and slip for the $2 \mathrm{D}$ static approximation correspond to

$$
f(x, t)=-\frac{\mu}{2 \pi \alpha} \mathrm{PV} \int_{-\infty}^{+\infty} \frac{1}{x-\xi} \delta_{, \xi}(\xi, t) \mathrm{d} \xi
$$

and emerge as long time limits of the present formulation. Here PV indicates the principal value, $\alpha=1$ for mode III and $\alpha=1-v$ for modes I and II, $v$ being Poisson's ratio. In the quasi-dynamic simulations by Rice (1993) for mode III, the radiation damping term in (1) is retained, so that solutions continue to exist through instabilities so that the integral in (38) is regarded as an approximation to $f(x, t)$.

Thus, similarly to Section 4 , representing $\delta$ as the Fourier series with period $2 L$, changing the domain of integration from $]-\infty,+\infty[$ to $[-L / 2,+L / 2]$, and then to $[x-L, x+L]$, and finally making the change of variable $u=x-\xi$ gives

$$
f(x, t)=-\frac{\mu}{2 \pi \alpha} \sum_{n=-\infty}^{+\infty} D_{n}(t) \frac{\mathrm{i} \pi n}{L} \mathrm{PV} \int_{-L}^{+L} \frac{\mathrm{e}^{-\mathrm{i} \pi n u / L}}{u} \mathrm{~d} u \mathrm{e}^{2 \mathrm{i} \pi n x / 2 L},
$$

which can be rewritten, after simplication, as

$$
f(x, t)=-\frac{\mu}{2 \alpha_{n}} \sum_{n=-\infty}^{+\infty} \frac{\pi|n|}{L}\left\{\frac{2}{\pi} \int_{0}^{|n| \pi} \frac{\sin u}{u} \mathrm{~d} u\right\} D_{n}(t) \mathrm{e}^{2 i \pi n x / 2 L}
$$

for $x \in[-L / 2,+L / 2]$. The integral within the curly brackets has to be computed numerically, its form showing that this can be done recursively for increasing $|n|$. For the original version of the spectral method, in which we would replace $2 L$ by $\lambda$ and accept a periodic replication of the crack with period $\lambda$, the same expression applies for $f(x, t)$ but with the curly bracket replaced by unity.

One can obtain a different formulation for the dynamic expression for $f(x, t)[(6)$ and (7)] by integrating the right-hand side of (7) by parts (Perrin et al., 1995), which results in the extraction of the static contribution of the stress and which is given by $f(x, t)$ in $(40)$ with the curly bracket replaced by unity, leaving a dynamic contribution in the form of the convolution of a "dynamic kernel" with the slip velocity (hence the terminology of "velocity formulation" by contrast to "displacement formulation" for the formulation so far developed). As the long time behavior is embodied in the static term, the convolution can be truncated when the truncated part has a negligible contribution, allowing both for computational gain and for mixing in a single com- 
putational procedure dynamic (unstable) rupture sequences with quasi-static ones without arbitrariness (Zheng et al., 1995; Rice and Ben-Zion, 1996).

To generalize the velocity formulation to the spectral method without replication, one has to integrate the right hand side of (16) by parts, $K(n, t)$ being given by (17). The resulting static term is precisely given by $(40)$ whereas the dynamic one involves a convolution kernel which has to be made explicit and computed similarly to what was done in Section 3 for the displacement formulation.

\section{GENERALIZATION TO THE THREE-DIMENSIONAL FORMULATION}

\subsection{Starting with the Fourier representation}

The conceptual simplicity of the spectral method comes from the fact that the 3D formulation is obtained rather directly from the $2 \mathrm{D}$ formulation, as shown by Geubelle and Rice (1995). In that situation, the various components $(j=x, y, z)$ of traction stresses $\tau_{j}$ and displacement discontinuities $\delta_{j}$ on the rupture plane $(y=0)$ are related by

$$
\tau_{j}(x, z, t)=\tau_{j}^{0}(x, z, t)-\Lambda_{j l} \delta_{l, t}(x, z, t)+f_{j}(x, z, t),
$$

where there is summation on the repeated $l$ over $x, y, z$, where $\Lambda_{j l}$ is diagonal with components $\left[\mu / 2 c_{\mathrm{s}},(\lambda+2 \mu) / 2 c_{\mathrm{d}}, \mu / 2 c_{\mathrm{s}}\right]$, and where each $f_{j}$ is a functional of the prior history of the $\delta_{l}$. In fact, for isotropic solids like those considered here, these partially decouple in that $f_{y}$ depends only on $\delta_{y}$ (mode I), whereas $f_{x}$ and $f_{z}$ each depend on both the $\delta_{x}$ and $\delta_{z}$ histories (shear modes). Indeed, from the Geubelle and Rice (1995) equations (32) and (34), we have

$$
\begin{aligned}
\tilde{f_{j}}(k, m, t) & =-\frac{\mu c_{\mathrm{s}}}{2} A_{j l}^{M}(k, m) \tilde{g}_{l M}(k, m, t), \\
\tilde{g}_{l M}(k, m, t) & =\int_{-\infty}^{t} C_{M}\left(q c_{\mathrm{s}}(t-\theta)\right) \tilde{\delta_{l}}(k, m, \theta) \mathrm{d} \theta,
\end{aligned}
$$

where there is summation over repeated indices $l$ and $M$, with $M$ summing over the modes I, II and III. The expressions for the convolution kernels $C_{M}$ are precisely those derived for the 2D formulation and repeated here in Appendix A. The twodimensional wave vector $\mathbf{q}=(k, m)$ spans the crack plane and we note that $q=|\mathbf{q}|$. Finally, $A_{j l}^{M}(k, m)$ are homogeneous quadratic terms in $k$ and $m$ that can be identified from Geubelle and Rice (1995) and are summarized in Appendix C.1. In (42), the tilde is intended for 2D Fourier transform, defined as

$$
\tilde{\delta_{l}}(k, m, \theta)=\int_{-\infty}^{+\infty} \int_{-\infty}^{+\infty} \delta_{l}\left(\xi_{1}, \xi_{3}, \theta\right) \mathrm{e}^{-\mathrm{i}\left(k \xi_{1}+m \xi_{3}\right)} \mathrm{d} \xi_{1} \mathrm{~d} \xi_{3} .
$$

Now we want to generalize the two-dimensional derivation of Section 3 to three dimensions, to avoid spatial replication. Let us note that we may want periodic replication of the rupture event along one direction of the rupture plane and avoid 
such replication along the other as in Rice and Ben-Zion (1996): they simulated earthquakes occurring in the earth's crust and so tried to reduce the effect of spatial replications along the vertical direction (or depth), but kept full spatial replication along the horizontal direction (or strike) so as to approximately represent an infinitely long seismic fault. Let us first assume that we are interested in a rupture confined to the region $x \in\left[-L_{1} / 2,+L_{1} / 2\right], z \in\left[-L_{3} / 2,+L_{3} / 2\right]$. We will thus perform the integration in the previous equation on these domains, then change to the domains $\left[x-L_{1}, x+L_{1}\right]$ in the $\xi_{1}$ direction and $\left[z-L_{3}, z+L_{3}\right]$ in the $\xi_{3}$ direction, and insert the Fourier series

$$
\delta_{l}\left(\xi_{1}, \xi_{3}, \theta\right)=\sum_{n_{1}=-\infty}^{+\infty} \sum_{n_{3}=-\infty}^{+\infty} D_{n_{1} n_{3}}^{I}(\theta) \mathrm{e}^{\mathrm{i}\left(h_{1} \xi_{1}+h_{3} \xi_{3}\right)},
$$

where $h_{1}=\pi n_{1} / L_{1}$ and $h_{3}=\pi n_{3} / L_{3}$, the periods of the series being $2 L_{1}$ and $2 L_{3}$ along the $x$ and $z$ axes, respectively. If, instead, we want to keep periodic replication along $x$ (say) and avoid it along $z$, then in (43) we will keep ] $-\infty,+\infty$ [ as the domain of integration for $\xi_{1}$ (i.e. taking $L_{1}=+\infty$ ) but take $h_{1}=2 \pi n_{1} / \lambda_{1}$ instead of $h_{1}=\pi n_{1} / L_{1}$ in the Fourier series; $\lambda_{1}$ will then be the replication period along $x$. Then we integrate over $\xi_{1}$ and $\xi_{3}$, and invert the Fourier transform to get $g(x, z, \theta)$ in a form that is valid for $x \in\left[-L_{1} / 2,+L_{1} / 2\right]$ and $z \in\left[-L_{3} / 2,+L_{3} / 2\right]$. Considering the first relation in (42) which is equivalent to

$$
f_{j}(x, z, t)=\frac{\mu c_{\mathrm{s}}}{2} A_{j l}^{M}\left(\frac{\partial}{\partial x}, \frac{\partial}{\partial z}\right) g_{l M}(x, z, t)
$$

we finally get

$$
f_{j}(x, z, t)=\sum_{n_{1}=-\infty}^{+\infty} \sum_{n_{3}=-\infty}^{+\infty} F_{n_{1} n_{3}}^{j^{*}}(t) \mathrm{e}^{\mathrm{i}\left(h_{1} x+h_{3} z\right)}
$$

where

$$
F_{n_{1} n_{3}}^{j^{*}}(t)=-\frac{\mu c_{\mathrm{s}}}{2} A_{j l}^{M}\left(h_{1}, h_{3}\right) \int_{-\infty}^{t} K_{M}\left(n_{1}, n_{3}, t-\theta\right) D_{n_{1} n_{3}}^{l}(\theta) \mathrm{d} \theta
$$

where in turn

$$
K_{M}\left(n_{1}, n_{3}, t\right)=\frac{1}{\pi^{2}} \int_{-\infty}^{+\infty} \int_{-\infty}^{+\infty} C_{M}\left(q c_{s} t\right) \frac{\sin L_{1}\left(k-h_{1}\right)}{k-h_{1}} \frac{\sin L_{3}\left(m-h_{3}\right)}{m-h_{3}} \mathrm{~d} k \mathrm{~d} m,
$$

with $q=\sqrt{k^{2}+m^{2}}$. The above formula is still valid for $L_{1}$ or $L_{3}$ infinite $:$ if, say, $L_{1} \rightarrow$ $+\infty, \sin L_{1}\left(k-h_{1}\right) /\left(k-h_{1}\right) \rightarrow \pi \delta_{\text {Dirac }}\left(k-h_{1}\right)$ and the formula can be simplified by integrating over $k$, giving the convolution kernel valid for the spectral method with periodic replication along $x$ only. [We can also check that if both $L_{1}$ and $L_{3}$ are infinite, we get the convolution kernel valid for the usual spectral method (i.e. with periodic replication along both directions): $K_{M}\left(n_{1}, n_{3}, t\right)=$ $C_{M}\left(\sqrt{\left(2 \pi n_{1} / \lambda_{1}\right)^{2}+\left(2 \pi n_{3} / \lambda_{3}\right)^{2}} c_{\mathrm{s}} t\right)$.] $K_{M}\left(n_{1}, n_{3}, t\right)$ as so defined can in principle be computed numerically, but the approach of the following sections gives a more suitable formula. 


\subsection{Spatio-temporal convolution in the three-dimensional case}

Let $G_{M}(x, z, t)$ be the function whose Fourier transform is $\widetilde{G}_{M}(k, m, t)=C_{M}\left(q c_{\mathrm{s}} t\right)$. Then using (42) and remembering that the product of Fourier transforms corresponds to spatial convolutions, the significance of $G_{M}(x, z, t)$ is that we may calculate the functions $f_{j}(x, z, t)$ by the space-time convolution

$f_{j}(x, z, t)=\frac{\mu c_{\mathrm{s}}}{2} \boldsymbol{A}_{j !}^{M}\left(\frac{\partial}{\partial x}, \frac{\partial}{\partial z}\right) \int_{-\infty}^{t} \int_{-\infty}^{+\infty} \int_{-\infty}^{+\infty} G_{M}\left(x-\xi_{1}, z-\xi_{3}, t-0\right)$

$$
\times \delta_{l}\left(\xi_{1}, \xi_{3}, \theta\right) \mathrm{d} \xi_{1} \mathrm{~d} \xi_{3} \mathrm{~d} \theta
$$

Now, we may note first that

$$
\int_{0}^{t} \tilde{G}_{M}(k, m, \tau) \mathrm{d} \tau=\frac{1}{q c_{\mathrm{s}}} \int_{0}^{q c_{\mathrm{s}} t} C_{M}\left(T^{\prime}\right) \mathrm{d} T^{\prime}
$$

and second, by the definition of $\mathscr{M}$ in (27) and some elementary calculations, that the integral on the right has the Laplace transform

$$
\int_{0}^{+\infty} \mathrm{e}^{-s T}\left[\int_{0}^{T} C_{M}\left(T^{\prime}\right) \mathrm{d} T^{\prime}\right] \mathrm{d} T=\frac{\mathscr{M}_{M}(s)}{s},
$$

where the functions $\mathscr{M}_{\mathrm{M}}$ are given in Appendix B. It is desirable to work with the integral on the left in (50) since, as well shall see, $G_{1}$ has a Dirac singularity at $r=c_{\mathrm{d}} t$ and $G_{\mathrm{II}}$ at $r=c_{\mathrm{s}} t$, where $r=\sqrt{x^{2}+z^{2}}$. To invert the Fourier transform of (50), we multiply by $(1 / 2 \pi)^{2} \exp [\mathrm{i}(k x+m z)]$ and integrate with $\mathrm{d} k \mathrm{~d} m$. Letting $\phi+\pi / 2$ be the angle between vectors $\mathbf{q}=(k, m)$ and $(x, z)$, we can write $k x+m z=-q r \sin \phi$ and use a polar representation to write $\mathrm{d} k \mathrm{~d} m=q \mathrm{~d} \phi \mathrm{d} q$. Thus

$$
\int_{0}^{l} G_{M}(x, z, \tau) \mathrm{d} \tau=\frac{1}{4 \pi^{2} c_{\mathrm{s}}} \int_{0}^{+\infty} \int_{-\pi}^{+\pi} \mathrm{e}^{-\mathrm{i} q r \sin \phi}\left[\int_{0}^{q c_{\mathrm{s}} t} C_{M}\left(T^{\prime}\right) \mathrm{d} T^{\prime}\right] \mathrm{d} \phi \mathrm{d} q .
$$

The integrals exist, if taken in this order, and indeed give a representation of the result as

$$
\int_{0}^{t} G_{M}(x, z, \tau) \mathrm{d} \tau=\frac{1}{2 \pi c_{3}} \int_{0}^{+\infty} J_{0}(q r)\left[\int_{0}^{q c_{s} t} C_{M}(T) \mathrm{d} T\right] \mathrm{d} q,
$$

where the integral on $q$ exists for all $r>0$ because the quantity in brackets has a finite limit [equal to $\mathscr{M}_{M}\left(0^{+}\right)$; see (27)] as $q \rightarrow+\infty$. However, it is more efficient to put a factor $\exp \left(-\varepsilon q c_{\mathrm{s}} t\right)$ into the integrand of $(52)$, where $\varepsilon>0$, and to interpret the result in the limit as $\varepsilon \rightarrow 0$. Then, interchanging the order of integration in (52), changing the $\phi$ range to $[-\pi / 2,+\pi / 2]$, and integrating on $T=q c_{s} t$ while using (51), and defining $R=r / c_{\mathrm{s}} t$,

$$
\begin{aligned}
\int_{0}^{t} G_{M}(x, z, \tau) \mathrm{d} \tau & =\frac{1}{2 \pi^{2} c_{s}^{2} t} \lim _{x \rightarrow 0} \int_{-\pi / 2}^{+\pi / 2} \frac{\mathscr{M}_{M}(\varepsilon+\mathrm{i} R \sin \phi)}{\varepsilon+\mathrm{i} R \sin \phi} \mathrm{d} \phi \\
& =\frac{1}{2 \pi^{2} c_{s}^{2} t}\left[\frac{\pi \mathscr{M}_{M}(+0)}{R}+\mathrm{PV} \int_{-\pi / 2}^{+\pi / 2} \frac{\mathscr{M}_{M}(\mathrm{i} R \sin \phi+0)}{\mathrm{i} R \sin \phi} \mathrm{d} \phi\right]
\end{aligned}
$$


For the present case, $\mathscr{M}_{M}(s)$ has complex conjugate $\overline{\mathscr{M}_{M}(s)}=\mathscr{M}_{M}(\bar{s})$, so the real part of $\mathscr{H}_{M}(\mathrm{i} R \sin \phi)$ is even in $\phi$, and hence contributes nothing to the integral. The imaginary part is odd in $\phi$ and hence $\operatorname{Im}[\mathscr{M}(\mathrm{i} R \sin \phi)] / \sin \phi$ is regular at $\phi=0$. We therefore obtain

$$
\int_{0}^{t} G_{M}(x, z, \tau) \mathrm{d} \tau=\frac{\mathscr{M}_{M}(0)}{2 \pi c_{\mathrm{s}} r}+\frac{1}{\pi^{2} c_{\mathrm{s}} r} \int_{0}^{\pi / 2} \frac{\operatorname{Im}\left[\mathscr{M}_{M}(\mathrm{i} R \sin \phi)\right]}{\sin \phi} \mathrm{d} \phi,
$$

where the limit of $\mathscr{M}_{M}(\mathrm{i} R \sin \phi)$ through positive real values is always understood.

Since $R \rightarrow 0$ as $t \rightarrow+\infty$ for fixed $r$, and since the integral involving $\mathscr{M}_{M}(\mathrm{i} R \sin \phi)$ vanishes in that limit, $\int_{0}^{t} G_{M}(x, z, \tau) \mathrm{d} \tau \rightarrow \mathscr{M}_{M}(0) / 2 \pi c_{s} r$. This result gives the static limit of the stress alteration due to some quiescent distribution of displacement discontinuity. Values of $\mathscr{M}_{M}(0)$ are noted in Appendix C.2.

The time dependence of $G_{M}(x, z, \tau)$ is given by

$$
G_{M}(x, z, t)=\frac{1}{\pi^{2} c_{\mathrm{s}} r} \frac{\partial}{\partial t} \int_{0}^{\pi / 2} \frac{\operatorname{Im}\left[\mathscr{M}_{M}\left(\mathrm{i}\left(r / c_{\mathrm{s}} t\right) \sin \phi\right)\right]}{\sin \phi} \mathrm{d} \phi
$$

and, as shown by the evaluation of the integrals in Appendix C.2, the integral has a discontinuity at $r=c_{\mathrm{d}} t$ for $M=\mathrm{I}$ and at $r=c_{\mathrm{s}} t$ for $M=\mathrm{II}$; those contribute Dirac singularities to $G_{\mathrm{I}}$ and $G_{\mathrm{II}}$, as indicated in (C.6). Recalling that $U(t)$ is the unit step function of time, with derivative $\dot{U}(t)=\delta_{\text {Dirac }}(t)$, we get

$$
\begin{aligned}
G_{\mathrm{I}}(x, z, t)= & \frac{-c_{\mathrm{d}}\left(1-2 c_{\mathrm{s}}^{2} / c_{\mathrm{d}}^{2}\right)^{2}}{2 \pi c_{\mathrm{s}}^{3} t} \delta_{\text {Dirac }}\left(t-\frac{r}{c_{\mathrm{d}}}\right)+\frac{c_{\mathrm{d}}}{2 \pi c_{\mathrm{s}}^{3} t^{2}} U\left(t-\frac{r}{c_{\mathrm{d}}}\right) \\
& -\frac{2 c_{\mathrm{s}} t}{\pi r^{3}}\left[U\left(t-\frac{r}{c_{\mathrm{d}}}\right)-U\left(t-\frac{r}{c_{\mathrm{s}}}\right)\right] \\
G_{\mathrm{II}}(x, z, t)= & \frac{-1}{2 \pi c_{\mathrm{s}}^{2} t} \delta_{\text {Dirac }}\left(t-\frac{r}{c_{\mathrm{s}}}\right)+\frac{1}{2 \pi c_{\mathrm{s}}^{2} t^{2}} U\left(t-\frac{r}{c_{\mathrm{s}}}\right) \\
& +\frac{2 c_{\mathrm{s}} t}{\pi r^{3}}\left[U\left(t-\frac{r}{c_{\mathrm{d}}}\right)-U\left(t-\frac{r}{c_{\mathrm{s}}}\right)\right] \\
G_{\mathrm{III}}(x, z, t)= & \frac{1}{2 \pi c_{\mathrm{s}}^{2} t^{2}} U\left(t-\frac{r}{c_{\mathrm{s}}}\right) .
\end{aligned}
$$

For the scalar, or model, elastodynamic theory as used by Rice et al. (1994) and Perrin and Rice (1994), and discussed in Appendix C.1, $G(x, z, t)=G_{\mathrm{III}}(x, z, t)$.

\subsection{Convolution kernels for the 3D spectral method without replication}

Let us finally use the expressions just derived for the spatio-temporal kernels $G_{M}$ to derive the convolution kernels for the three-dimensional method without replication, 
similarly to what has been done in Section 4.2 for the two-dimensional case. We begin with (49). The rupture has non-zero $\delta_{l}\left(\xi_{1}, \xi_{3}, 0\right)$ for $\left.\xi_{1} \in\right]-L_{1} / 2,+L_{1} / 2\left[, \xi_{3} \in\right]-L_{3} / 2,+L_{3} / 2[$ so that in (49) we want to integrate over this domain instead of over the whole plane. Then, changing the ranges of integration to $\left[x-L_{1}, x+L_{1}\right]$ and $\left[z-L_{3}, z+L_{3}\right]$, inserting the Fourier series representation (44) of $\delta_{l}\left(\xi_{1}, \xi_{3}, \theta\right)$ and making the changes of variables $u_{1}=x-\xi_{1}$ and $u_{3}=z-\xi_{3}$, we get :

$$
\begin{aligned}
f_{i}(x, z, t)=-\frac{\mu c_{\mathrm{s}}}{2} & \sum_{n_{1}=-\infty}^{+\infty} \sum_{n_{3}=-\infty}^{+\infty} A_{j l}^{M}\left(h_{1}, h_{3}\right) \int_{-\infty}^{t} D_{n_{1} n_{3}}^{l}(\theta) \\
& \times \int_{-L_{1}}^{L_{i}} \int_{-L_{3}}^{L_{3}} G_{M}\left(u_{1}, u_{3}, t-\theta\right) \mathrm{e}^{-\mathrm{i}\left(h_{1} u_{1}+h_{3} u_{3}\right)} \mathrm{d} u_{1} \mathrm{~d} u_{3} \mathrm{~d} \theta \mathrm{e}^{\mathrm{i}\left(h_{1} x+h_{3} z\right)},
\end{aligned}
$$

which is valid for $x \in\left[-L_{1} / 2,+L_{1} / 2\right]$ and $z \in\left[-L_{3} / 2,+L_{3} / 2\right]$. [We recall that we allow the possibility for $L_{1}$ (resp. $L_{3}$ ) to be $+\infty$, thus keeping spatial replication along $x$ (resp. $z$ ). In this case, in the Fourier series, $h_{1}=2 \pi n_{1} / \lambda_{1}$ (resp. $h_{3}=2 \pi n_{3} / \lambda_{3}$ ), $\lambda_{1}$ (resp. $\hat{\lambda}_{3}$ ) being the replication period along $x$ (resp. $z$ ).] This is a Fourier series in the form of (46) and (47), where the innermost integrals provide the expression for $K_{M}\left(n_{1}, n_{3}, t\right)$ as

$$
K_{M}\left(n_{1}, n_{3}, t\right)=\int_{-L_{1}}^{L_{1}} \int_{-L_{3}}^{L_{3}} G_{M}\left(u_{1}, u_{3}, t\right) \mathrm{e}^{-\mathrm{i}\left(h_{1} u_{1}+h_{3} u_{3}\right)} \mathrm{d} u_{1} \mathrm{~d} u_{3} .
$$

It is much more natural and easier to compute these integrals in polar coordinates. Then, taking symmetries into account, we can check that, as expected, the $K_{M}$ are real and that we therefore have

$$
K_{M}\left(n_{1}, n_{3}, t\right)=2 \int_{\psi q}^{\psi_{q}+\pi} \int_{0}^{\Re(\psi)} G_{M}(r, t) \cos \left[r\left(h_{1} \cos \psi+h_{3} \sin \psi\right)\right] r \mathrm{~d} r \mathrm{~d} \psi,
$$

where $\psi_{q}$ can be chosen arbitrarily, and for expressions below is chosen on $[-\pi / 2,0]$, $\tan \psi=u_{3} / u_{1}, \mathscr{R}(\psi)$ is the distance from the origin to the rectangular boundary (see Fig. 5) and $r=\sqrt{u_{1}^{2}+u_{3}^{2}}$. We must now evaluate the expressions for $K_{M}$ for the particular expressions for $G_{M}$. Equations (57) show that this involves three terms: $G^{(a)}(r, t)=\delta_{\text {Dirac }}(t-r / c), G^{(b)}(r, t)=U(t-r / c)$ and $G^{(c)}(r, t)=U(t-r / c) r^{3}, c$ denoting $c_{\mathrm{s}}$ or $c_{\mathrm{d}}$. Integration over $r$ is straightforward to perform. Noting $K^{(i)}(c)$, the terms corresponding to $G^{(i)}(i=\mathrm{a}, \mathrm{b}, \mathrm{c})$, we have

$$
\begin{aligned}
K^{(\mathrm{a})}(c) & =2 c^{2} t \int_{\psi_{q}}^{\psi_{q}+\pi} U(\mathscr{R}(\psi)-c t) \cos [B(c t, \psi)] \mathrm{d} \psi, \\
K^{(\mathrm{b})}(c) & =\int_{\psi_{q}}^{\psi_{q}+\pi} C(\min [c t, \mathscr{R}(\psi)], \psi) \mathrm{d} \psi, \\
K^{(\mathrm{c})}(c) & =\int_{\psi_{q}}^{\psi_{q}+\pi} D(\min [c t, \mathscr{R}(\psi)], \psi) \mathrm{d} \psi,
\end{aligned}
$$

where we define 


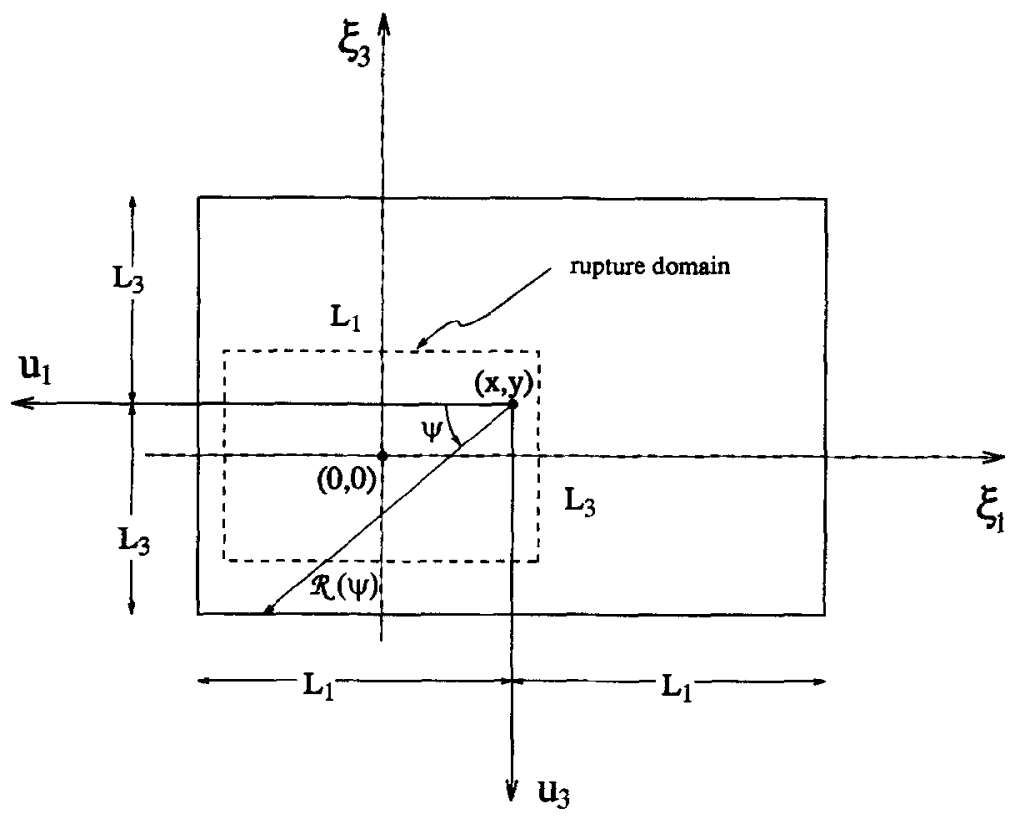

Fig. 5. Rupture domain (inside dashed rectangle) and integration domain (inside full line rectangle) of the function whose integral gives the convolution kernels for the spectral method without replication in three dimensions.

$$
\begin{aligned}
B(r, \psi) & =r\left(h_{1} \cos \psi+h_{3} \sin \psi\right), \\
C(r, \psi) & =\frac{2 r^{2}(\cos [B(r, \psi)]-1)}{[B(r, \psi)]^{2}}+\frac{2 r^{2} \sin [B(r, \psi)]}{B(r, \psi)}, \\
D(r, \psi) & =\frac{-2 \cos [B(r, \psi)]}{r}-\frac{2 B(r, \psi) \operatorname{Si}[B(r, \psi)]}{r}, \\
\text { Si }(x) & =\text { sine integral }=\int_{0}^{x} \frac{\sin t}{t} \mathrm{~d} t .
\end{aligned}
$$

Note that $K^{(\mathrm{c})}$ is not obtained, as for $K^{(\mathrm{a})}$ and $K^{(\mathrm{b})}$, by inserting $G^{(\mathrm{c})}(r, t)$ into $(60)$ (since it would not be defined). Instead, one must integrate on $r$ the term depending on $c_{\mathrm{s}}$ together with the one depending on $c_{\mathrm{d}}$. We have:

$$
\begin{aligned}
K_{\mathrm{l}}\left(n_{1}, n_{3}, t\right) & =\frac{-c_{\mathrm{d}}\left(\mathrm{l}-2 c_{\mathrm{s}}^{2} / c_{\mathrm{d}}^{2}\right)^{2}}{2 \pi c_{\mathrm{s}}^{3} t} K^{(\mathrm{a})}\left(c_{\mathrm{d}}\right)+\frac{c_{\mathrm{d}}}{2 \pi c_{\mathrm{s}}^{3} t^{2}} K^{(\mathrm{b})}\left(c_{\mathrm{d}}\right)-\frac{2 c_{\mathrm{s}} t}{\pi}\left[K^{(\mathrm{c})}\left(c_{\mathrm{d}}\right)-K^{(\mathrm{c})}\left(c_{\mathrm{s}}\right)\right], \\
K_{\mathrm{II}}\left(n_{1}, n_{3}, t\right) & =\frac{-1}{2 \pi c_{\mathrm{s}}^{2} t} K^{(\mathrm{a})}\left(c_{\mathrm{s}}\right)+\frac{1}{2 \pi c_{\mathrm{s}}^{2} t^{2}} K^{(\mathrm{b})}\left(c_{\mathrm{s}}\right)+\frac{2 c_{\mathrm{s}} t}{\pi}\left[K^{(\mathrm{c})}\left(c_{\mathrm{d}}\right)-K^{(\mathrm{c})}\left(c_{\mathrm{s}}\right)\right] \\
K_{\mathrm{III}}\left(n_{1}, n_{3}, t\right) & =\frac{1}{2 \pi c_{\mathrm{s}}^{2} t^{2}} K^{(\mathrm{b})}\left(c_{\mathrm{s}}\right)
\end{aligned}
$$


where the $K^{(i)}(c)$ are given in Appendix C.3, for the different possible values of $c t$ with respect to $L_{1}, L_{3}$, and $\sqrt{L_{1}^{2}+L_{3}^{2}}$.

Those formulae are valid for infinite $L_{1}$ (resp. $L_{3}$ ), which corresponds to the case where we want spatial replication along $x$ (resp. $z$ ) only. We can check that, if $c_{\mathrm{d}} t<\mathscr{R}(\psi)$ for every $\psi$ (i.e. for a small enough time or if $L_{1}$ and $L_{3}$ are both infinite), $K_{M}\left(n_{1}, n_{3}, t\right)=C_{M}\left(q_{13} c_{s} t\right)$ with $q_{13}=\sqrt{h_{1}^{2}+h_{3}^{2}}$ and where the $C_{M}(T)$, given in Appen$\operatorname{dix} \mathrm{A}$, are the convolution kernels for the spectral method with spatial replication.

We can also see that $K_{M}\left(n_{1}, n_{3}, t\right)$ is zero when $t$ is equal to or greater than $t_{\mathrm{c}}=\sqrt{L_{1}^{2}+L_{3}^{2}} / c_{\mathrm{s}}$ (since, for those times, $K^{(\mathrm{a})}=K^{(\mathrm{b})}=0$ and $K^{(\mathrm{c})}(c)$ does not depend on the velocity ()$, t_{\mathrm{c}}$ being the time requircd by the shear wave to cross the largest rupture domain dimension, i.e., the static limit of the kernel is obtained for $t>t_{\mathrm{c}}$. This property, specific to $3 \mathrm{D}$ problems (contrary to $2 \mathrm{D}$ problems for which the static limit is reached asymptotically, i.e., in infinite time), allows for substantial computation gain: the time convolution involved in the evaluation of the functional $f$ can be written as

$$
\int_{-\infty}^{t} K_{M}\left(n_{1}, n_{3}, t-\theta\right) D_{n_{1} n_{3}}^{\prime}(\theta) \mathrm{d} \theta=\int_{t-t_{\mathrm{c}}}^{t} K_{M}\left(n_{1}, n_{3}, t-\theta\right) D_{n_{1} n_{3}}^{\prime}(\theta) \mathrm{d} \theta .
$$

\section{ACKNOWLEDGEMENTS}

We thank Yehuda Ben-Zion, Daniel Fisher, Philippe Geubelle, John Morrissey and Gutuan Zheng for useful discussions. The research was supported by the Office of Naval Research, Solid Mechanics Program, through grant number N00014-96-10777, and the NSF Southern California Earthquake Center through subcontract PO-621911 from the University of Southern California. Support for access to a CM-5 computer has been provided by NSF grant EAR940004N through NCSA.

\section{REFERENCES}

Bouchon, M. and Aki, K. (1977) Discrete wave-number representation of seismic source wave fields. Bull. Seismol. Soc. Am. 67(2), 259-277.

Bouchon, M. and Streiff, D. (1997) Propagation of a shear crack on a non-planar fault : a method of calculation. Bull. Seismol. Soc. Am. 87(1), 61-66.

Burridge, R. (1969) The numerical solution of certain integral equations with non-integrable kernels arising in the theory of crack propagation and elastic wave diffraction. Phil. Trans. R. Soc. A265, 353-381.

Cochard, A. and Madariaga, R. (1994) Dynamic faulting under rate-dependent friction. Pure Appl. Geophys. 142, 419-445.

Geubelle, P. H. and Rice, J. R. (1995) A spectral method for three-dimensional elastodynamic fracture problems. J. Mech. Phys. Solids 43(11), 1791-1824.

Geubelle, P. H., Danyluk, M. J. and Hilton, H. H. (1997) Dynamic mode 3 fracture in viscoelastic media. Int. J. Solids Struct., in press.

Kostrov, B. V. (1966) Unsteady propagation of longitudinal shear cracks. J. Appl. Math. Mech. 30, 1241-1248.

Morrissey, J. M. and Geubelle, P. H. (1997) A numerical scheme for mode III dynamic fracture problems. Int. J. Numer. Methods Engng 40, 1181-1196. 
Perrin, G. and Rice, J. R. (1994) Disordering of a dynamic planar crack front in a model elastic medium of randomly variable toughness. J. Mech. Phys. Solids 42(6), 1047-1064.

Perrin, G., Rice, J. R. and Zheng, G. (1995) Self-healing slip pulse on a frictional surface. $J$. Mech. Phys. Solids 43(9), 1461-1495.

Rice, J. R. (1993) Spatio-temporal complexity of slip on a fault. J. Geophys. Res. 98, 98859907.

Rice, J. R. and Ben-Zion, Y. (1996) Slip complexity in earthquake fault models. Proc. Natl. Acad. Sci. U.S.A. 93(9), 3811-3818.

Rice, J. R., Ben-Zion, Y. and Kim, K.-S. (1994) Three-dimensional perturbation solution for a dynamic planar crack moving unsteadily in a model elastic solid. J. Mech. Phys. Solids 42, 813-843.

Willis, J. R. (1973) Self-similar problems in elastodynamics. Phil. Trans. R. Soc. A274, 435491.

Willis, J. R. and Movchan, A. B. (1995) Dynamic weight functions for a moving crack. I. Mode I loading. J. Mech. Phys. Solids 43(3), 319-341.

Wolfram, S. (1993) Mathematica: A System for Doing Mathematics by Computer. AddisonWesley, Reading, MA.

Zheng, G., Ben-Zion, Rice, J. R. and Morrissey, J. (1995) A new method for calculating elastodynamic response over long stressing histories containing rapid rupture episodes. EOS Trans. Am. Geophys. Union (Fall Meeting Supplement) 76(46), 405.

\section{APPENDIX A : CONVOLUTION KERNELS IN THE FOURIER DOMAIN}

This appendix gives the expression for the convolution kernels in the Fourier domain for each mode. They have been derived in Geubelle and Rice (1995) for modes I and II and in Perrin et al. (1995) for mode III :

$$
\begin{aligned}
C_{\mathrm{I}}(T) & =\left(\frac{c_{\mathrm{d}}}{c_{\mathrm{s}}}\right)^{3} \frac{J_{\mathrm{l}}\left(c_{\mathrm{d}} T / c_{\mathrm{s}}\right)}{c_{\mathrm{d}} T / c_{\mathrm{s}}}+4 T\left[W(T)-W\left(c_{\mathrm{d}} T / c_{\mathrm{s}}\right)\right]+\left[4 \frac{c_{\mathrm{d}}}{c_{\mathrm{s}}}-\left(\frac{c_{\mathrm{d}}}{c_{\mathrm{s}}}\right)^{3}\right] J_{0}\left(c_{\mathrm{d}} T / c_{\mathrm{s}}\right)-4 J_{0}(T), \\
C_{\mathrm{II}}(T) & =J_{1}(T) / T+4 T\left[W\left(c_{\mathrm{d}} T / c_{\mathrm{s}}\right)-W(T)\right]-4 \frac{c_{\mathrm{s}}}{c_{\mathrm{d}}} J_{0}\left(c_{\mathrm{d}} T / c_{\mathrm{s}}\right)+3 J_{0}(T), \\
C_{\mathrm{III}}(T) & =J_{1}(T) / T
\end{aligned}
$$

where $J_{0}(T)$ and $J_{1}(T)$ are Bessel functions and

$$
W(T)=1-\int_{0}^{T} \frac{J_{1}(x)}{x} \mathrm{~d} x .
$$

[The factor $-4 c_{\mathrm{s}} / c_{\mathrm{d}}$ in $C_{\mathrm{II}}(T)$ has been wrongly written $-4 c_{\mathrm{d}} / c_{\mathrm{s}}$ in equation (26) of Geubelle and Rice (1995); also, in their Fig. 2, the scale along the $T$ axis for $C_{\mathrm{I}}(T)$ and $C_{\mathrm{II}}(T)$ is not correct and should instead range from 0 to approximately 47 ; finally, still in that figure, the labels $F_{1}, F_{2}, F_{3}$ should read, respectively, $C_{1}, C_{\mathrm{II}}, C_{\mathrm{III}}$.]

\section{APPENDIX B : CONVOLUTION KERNELS IN THE SPATIO-TEMPORAL DOMAIN}

The expressions for the functions $\mathscr{M}_{M}(s)$ for the three modes are (Geubelle and Rice, 1995):

$$
\mathscr{M}_{1}(s)=-\frac{4 \sqrt{s^{2}+1}}{s^{2}}+\frac{\left(2+s^{2}\right)^{2}}{s^{2} \sqrt{\gamma^{2} s^{2}+1}}-\frac{s}{\gamma},
$$




$$
\begin{aligned}
& \mathscr{M}_{\mathrm{II}}(s)=\frac{\left(2+s^{2}\right)^{2}}{s^{2} \sqrt{s^{2}+1}}-\frac{4 \sqrt{\gamma^{2} s^{2}+1}}{s^{2}}-s, \\
& \mathscr{M}_{\mathrm{III}}(s)=\sqrt{s^{2}+1}-s
\end{aligned}
$$

with $\sqrt{s^{2}+1}$ and $\sqrt{\gamma^{2} s^{2}+1}$ cut on the imaginary $s$ axis to behave like $s$ and $\gamma s$, respectively, at large $s$; here $\gamma=c_{\mathrm{s}} / c_{\mathrm{d}}$. Thus the function $M(u)=\operatorname{Re}[\mathscr{M}(\mathrm{i} u+0)]$ of (32) and (36) are

$$
\begin{aligned}
& M_{\mathrm{I}}(u)= \begin{cases}\frac{4 \sqrt{1-u^{2}} \sqrt{1-\left(c_{\mathrm{s}}^{2} / c_{\mathrm{d}}^{2}\right) u^{2}}-\left(2-u^{2}\right)^{2}}{u^{2} \sqrt{1-\left(c_{\mathrm{s}}^{2} / c_{\mathrm{d}}^{2}\right) u^{2}}} & \text { if }|u|<1, \\
\frac{-\left(2-u^{2}\right)^{2}}{u^{2} \sqrt{1-\left(c_{\mathrm{s}}^{2} / c_{\mathrm{d}}^{2}\right) u^{2}}} & \text { if } 1<|u|<c_{\mathrm{d}} / c_{\mathrm{s}}, \\
0 & \text { if }|u|>c_{\mathrm{d}} / c_{\mathrm{s}},\end{cases} \\
& M_{\mathrm{II}}(u)= \begin{cases}\frac{4 \sqrt{1-u^{2}} \sqrt{1-\left(c_{\mathrm{s}}^{2} / c_{\mathrm{d}}^{2}\right) u^{2}}-\left(2-u^{2}\right)^{2}}{u^{2} \sqrt{1-u^{2}}} & \text { if }|u|<1, \\
\frac{4 \sqrt{1-\left(c_{\mathrm{s}}^{2} / c_{\mathrm{d}}^{2}\right) u^{2}}}{u^{2}} & \text { if } 1<|u|<c_{\mathrm{d}} / c_{\mathrm{s}}, \\
0 & \text { if }|u|>c_{\mathrm{d}} / c_{\mathrm{s}},\end{cases} \\
& M_{\mathrm{III}}(u)= \begin{cases}\sqrt{1-u^{2}} & \text { if }|u|<1, \\
0 & \text { if }|u|>1 .\end{cases}
\end{aligned}
$$

\section{APPENDIX C : THREE-DIMENSIONAL EXPRESSIONS}

\section{C.1. Quadratic forms}

The quadratic forms $A_{j l}^{M}(k, m)$ enter (45). Of these, $A_{j l}^{1}=0$ except when $j=l=y$, in which case

$$
A_{y y}^{1}=q^{2}=k^{2}+m^{2}
$$

Also, $A_{j l}^{\mathrm{II}}=\mathbf{0}$ and $A_{j l}^{\mathrm{III}}=0$ when one or both of $j$ and $l$ is $y$. The non-zero members are

$$
\left[\begin{array}{cc}
A_{x x}^{\mathrm{II}} & A_{x z}^{\mathrm{II}} \\
A_{z x}^{\mathrm{II}} & A_{z z}^{\mathrm{II}}
\end{array}\right]=\left[\begin{array}{ll}
k^{2} & m k \\
m k & m^{3}
\end{array}\right],\left[\begin{array}{cc}
A_{x x}^{\mathrm{III}} & A_{x z}^{\mathrm{III}} \\
A_{z x}^{\mathrm{III}} & A_{z z}^{\mathrm{III}}
\end{array}\right]=\left[\begin{array}{cc}
m^{2} & -m k \\
-m k & k^{2}
\end{array}\right] .
$$

Another case of interest is for the "model" elastodynamic theory used by Rice et al. (1994) and Perrin and Rice (1994). This is based on a scalar wave equation, and in that case there are no components $j, l$ or modes $M$ to sum over in the relation between stress and displacement. With such indices removed, the equation here describes the case where $\mu$ and $c_{\mathrm{s}}$ are interpreted as the modulus and wave speed [called $M$ and $c$ in Rice $e t$ al. (1994)] of the model theory. Then the radiation damping factor is $\Lambda=\mu / 2 c_{\mathrm{s}}, A(k, m)=k^{2}+m^{2}, \quad C(T)=C_{\mathrm{III}}(T)$ and $\mathscr{M}(s)=\mathscr{M}_{\text {III }}(s)$. 
C.2. Three-dimensional space-time convolution functions

To evaluate the functions $G_{M}(x, z, t)$ of (55), we first note that $\mathscr{M}_{\mathrm{I}}(+0)=$ $\mathscr{M}_{\mathrm{II}}(+0)=2\left(1-\gamma^{2}\right)$, and $\mathscr{M}_{\mathrm{III}}(+0)=1$. Here $\gamma=c_{\mathrm{s}} / c_{\mathrm{d}}$. Next we must evaluate the integrals

$$
Q_{M}(R)=\int_{0}^{\pi / 2} \frac{\operatorname{Im}\left[\mathscr{M}_{M}(\mathrm{i} R \sin \phi+0)\right]}{\sin \phi} \mathrm{d} \phi
$$

appearing in (55). The imaginary parts of $\mathscr{M}_{M}(\mathrm{i} u)\left[=\mathscr{M}_{M}(\mathrm{i} u+0)\right]$ are :

$$
\begin{aligned}
& \operatorname{Im}\left[\mathscr{M}_{1}(\mathrm{i} u+0)\right]= \begin{cases}-u / \gamma & \text { if } 0<u<1, \\
-u / \gamma+4 \sqrt{u^{2}-1} / u^{2} & \text { if } 1<u<1 / \gamma, \\
-u / \gamma+4 \sqrt{u^{2}-1} / u^{2}+\left(2-u^{2}\right)^{2} / u^{2} \sqrt{\gamma^{2} u^{2}-1} & \text { if } u>1 / \gamma,\end{cases} \\
& \operatorname{Im}\left[\mathscr{M}_{\mathrm{II}}(\mathrm{i} u+0)\right]= \begin{cases}-u & \text { if } 0<u<1, \\
-u+\left(2-u^{2}\right)^{2} / u^{2} \sqrt{u^{2}-1} & \text { if } 1<u<1 / \gamma, \\
-u+\left(2-u^{2}\right)^{2} / u^{2} \sqrt{u^{2}-1}+4 \sqrt{\gamma^{2} u^{2}-1} / u^{2} & \text { if } u>1 / \gamma,\end{cases} \\
& \operatorname{Im}\left[\mathscr{M}_{\mathrm{III}}(\mathrm{i} u+0)\right]= \begin{cases}-u & \text { if } 0<u<1, \\
-u+\sqrt{u^{2}-1} & \text { if } u>1 .\end{cases}
\end{aligned}
$$

Let us write $Q_{M}(R)=Q_{M}^{(1)}(R)+Q_{M}^{(2)}(R)+Q_{M}^{(3)}(R)$, where $Q_{M}^{(1)}(R)$ comes from the first term in $\operatorname{Im}\left[\mathscr{M}_{M}(\mathrm{i} u)\right]$, equal or proportional to $-u$, which enters at $u=0$ and persists for all $u>0$, $Q_{M}^{(2)}(R)$ describes the effect of the new terms that enter $\operatorname{Im}\left[\mathscr{M}_{M}(\mathrm{i} u)\right]$ at $u=1$ and persist for all $u>1$, and $Q_{M}^{(3)}(R)$ describes the effect of terms that enter $\operatorname{Im}\left[\mathscr{M}_{M}(\mathrm{i} u)\right]$ at $u=1 / \gamma$ and persist for $u>1 / \gamma=c_{\mathrm{d}} / c_{\mathrm{s}}$. Thus $Q_{M}^{(2)}(R)=0$ for $R<1, Q_{M}^{(3)}(R)=0$ for $R<1 / \gamma ; Q_{\text {iI }}^{(3)}(R) \equiv 0$.

Causality requires that all $G_{M}=0$ for $t<r / c_{\mathrm{d}}$, i.e. for $R>1 / \gamma$, which means that the $Q_{M}^{(i)}(R)$ should sum to a constant in that range, a condition which may be explicitly checked. The constant can be obtained from (55), which thus must vanish for $R>1 / \gamma$, so that $Q_{M}^{(3)}(R)$ for $R>1 / \gamma$ can be determined (or checked) from

$$
Q_{M}^{(1)}(R)+Q_{M}^{(2)}(R)+Q_{M}^{(3)}(R)+(\pi / 2) \mathscr{M}_{M}(0)=0, \quad R>1 / \gamma
$$

Some of the $Q M(R)$ start from non-zero values on their range $R$ of definition, which makes $Q_{M}(R)$ discontinuous and puts Dirac singularities in (56). Remembering that increasing $R$ means decreasing $t$ at fixed $r$, we therefore have from (56) that

$$
\begin{aligned}
G_{M}(x, z, t)=-\frac{1}{\pi^{2} c_{\mathrm{s}}^{2} t^{2}} & \frac{\mathrm{d} Q_{M}^{(1)}}{\mathrm{d} R} U\left(t-r / c_{\mathrm{s}}\right) \\
& -\frac{1}{\pi^{2} c_{\mathrm{s}}^{2} t^{2}}\left[\frac{\mathrm{d} Q_{M}^{(1)}}{\mathrm{d} R}+\frac{\mathrm{d} Q_{M}^{(2)}}{\mathrm{d} R}\right]\left[U\left(t-r / c_{\mathrm{d}}\right)-U\left(t-r / c_{\mathrm{s}}\right)\right] \\
& -\frac{1}{\pi^{2} c_{\mathrm{s}}^{2} t} Q_{M}^{(2)}(1) \delta_{\text {Dirac }}\left(t-r / c_{\mathrm{s}}\right)-\frac{1}{\pi^{2} c_{\mathrm{s}} c_{\mathrm{d}} t} Q_{M}^{(3)}(1 / \gamma) \delta_{\text {Dirac }}\left(t-r / c_{\mathrm{d}}\right) .
\end{aligned}
$$

The last three terms are absent for the mode III part of the solution, $G_{\mathrm{III}}$, and also for $G$ for the scalar "model" elastic theory mentioned above.

The $Q_{M}^{(1)}(R)$ are defined for $R>0$ and calculated from the part $\operatorname{Im}\left[\mathscr{H}_{M}(\mathrm{i} u)\right]=-u$ for $M=\mathrm{II}$, III, and $-u / \gamma$ for $M=\mathrm{I}$, so that

$$
Q_{1}^{(1)}(R)=-\pi R / 2 \gamma, \quad Q_{\mathrm{II}}^{(1)}(R)=Q_{\mathrm{III}}^{(1)}(R)=-\pi R \therefore
$$


The terms entering $\operatorname{Im}\left[\mathscr{M}_{M}(\mathrm{i} u)\right]$ when $u>1$, and defining $Q_{M}^{(2)}(R)$ for $R>1$, contain a factor $\sqrt{u^{2}-1}$. We write those terms as $u^{2} h_{M}\left(u^{2}\right) / \sqrt{u^{2}-1}$ where

$$
h_{\mathrm{I}}(p)=4(p-1) / p^{2}, \quad h_{\mathrm{II}}(p)=1-4(p-1) / p^{2}, \quad h_{\mathrm{III}}(p)=(p-1) / p .
$$

Thus

$$
Q_{M}^{(2)}(R)=\int_{\arcsin (1 / R)}^{\pi / 2} \frac{\left(R^{2} \sin ^{2} \phi\right) h_{M}\left(R^{2} \sin ^{2} \phi\right)}{\sin \phi \sqrt{R^{2} \sin ^{2} \phi-1}} \mathrm{~d} \phi .
$$

Making the change of variable $R \cos \phi=\sqrt{R^{2}-1} \sin (\omega / 2)$ then leads to

$$
Q_{M}^{(2)}(R)=\frac{R}{2} \int_{0}^{\pi} h_{M}\left(\frac{R^{2}+1}{2}+\frac{R^{2}-1}{2} \cos \omega\right) \mathrm{d} \omega,
$$

which can be extended to $[0,2 \pi]$, have its integration variable changed to $\sigma=\exp (i \omega)$, and evaluated by methods of analytic function theory (residues). Thus we obtain

$$
Q_{\mathrm{I}}^{(2)}(R)=\pi\left(1-1 / R^{2}\right), \quad Q_{\mathrm{11}}^{(2)}(R)=\pi R / 2-\pi\left(1-1 / R^{2}\right), \quad Q_{\mathrm{ni1}}^{(2)}(R)=\pi(R-1) / 2 .
$$

Note that $Q_{M}^{(2)}(1)$ is non-zero, thus corresponding to a Dirac function at $r=\mathrm{c}_{\mathrm{s}} t$ in $G_{M}$, only for $M=$ II. We also need $Q_{M}^{(3)}(1 / \gamma)$ to give the amplitude of Dirac functions at $r=c_{\mathrm{d}} t$. By using (C. 5$)$,

$$
Q_{M}^{(3)}(1 / \gamma)=-Q_{M}^{(1)}(1 / \gamma)-Q_{M}^{(2)}(1 / \gamma)-\pi \mathscr{M}_{M}(0) / 2
$$

This is relevant only for $M=1, \mathrm{II}$ and we find

$$
Q_{1}^{(3)}(1 / \gamma)=\frac{\pi}{2 \gamma^{2}}\left(1-2 \gamma^{2}\right)^{2}, \quad Q_{11}^{(3)}(1 / \gamma)=0
$$

\section{C.3. Further details}

Letting $L_{\min }=\min \left[L_{1}, L_{3}\right], L_{\max }=\max \left[L_{1}, L_{3}\right]$ and

$$
\psi_{\alpha}=\arcsin \left(\frac{L_{\min }}{c t}\right), \quad \psi_{\beta}=\operatorname{arcos}\left(\frac{L_{\max }}{c t}\right), \quad \psi_{\eta}=\arctan \left(\frac{L_{\min }}{L_{\max }}\right),
$$

all confined to $[0, \pi / 2]$, and defining the functions $S(\psi)$ and $T(\psi)$ such that

$$
\frac{\mathrm{d} S(\psi)}{\mathrm{d} \psi}=C\left(\frac{L_{\min }}{\sin \psi}, \psi\right), \quad \frac{\mathrm{d} T(\psi)}{\mathrm{d} \psi}=C\left(\frac{L_{\max }}{\cos \psi}, \psi\right),
$$

$S$ and $T$ having been determined using the Mathematica software (Wolfram, 1993) as

$$
\begin{aligned}
& S(\psi)=\frac{-4 \sin \psi}{B\left(h_{1}, \psi\right)} \sin ^{2}\left[B\left(\frac{L_{\min }}{2 \sin \psi}, \psi\right)\right], \\
& T(\psi)=\frac{4 \cos \psi}{B\left(h_{3}, \psi\right)} \sin ^{2}\left[B\left(\frac{L_{\max }}{2 \cos \psi}, \psi\right)\right],
\end{aligned}
$$

we finally have the following, assuming $L_{\max }=L_{1}: \dagger$

$\uparrow$ For the expression to be valid if $L_{\max }=L_{3}$, one has to replace $\psi_{x}, \psi_{\beta}, \psi_{\gamma}$ by $\psi_{\alpha}+\pi / 2, \psi_{\beta}+\pi / 2, \psi_{\gamma}+\pi / 2$, respectively. 
if $0 \leqslant c t<L_{\min }$

$$
\begin{aligned}
& K^{(\mathrm{a})}(c)=2 \pi c^{2} t J_{0}\left(q_{13} c t\right), \\
& K^{(\mathrm{b})}(c)=2 \pi(c t)^{2} J_{1}\left(q_{13} c t\right) /\left(q_{13} c t\right), \\
& K^{(c)}(c)=2 \pi q_{13}\left[W\left(q_{13} c t\right)-J_{0}\left(q_{13} c t\right) /\left(q_{13} c t\right)-1\right],
\end{aligned}
$$

with $q_{13}=\sqrt{h_{1}^{2}+h_{3}^{2}}$. The first two equalities above are deduced rather directly from the definition of $K^{(a)}$ and $K^{(b)}$ in (61). As for the third, one can check that $K^{(\text {c) }}$ and $2 \pi q_{13} W\left(\mathrm{q}_{13} c t\right)-2 \pi J_{0}\left(q_{13} c t\right) /\left(q_{13} c t\right)$ obey the same differential equation $d^{2} X / \mathrm{d} q_{13}^{2}=\left(2 \pi / q_{13}\right) J_{0}^{\prime}\left(q_{13} c t\right)$, so that they differ only be an extra term which either does not depend on $q_{13}$ or depends on it at first order. Then, as $\int_{0}^{2 \pi} \cos [B(c t, \psi)] / c t \mathrm{~d} \psi=\pi J_{0}\left(q_{13} c t\right) / c t$, and $\int_{0}^{2 \pi} B(c t, \psi) \operatorname{Si}[B(c t, \psi)] / c t \mathrm{~d} \psi=0$ for $c t=0$, we find the extra term $2 \pi q_{13}\left[=2 \pi q_{13} W\left(q_{13} \times 0\right)\right]$;

if $L_{\min } \leqslant c t<L_{\max }$,

$$
\begin{aligned}
& K^{(\mathrm{a})}(c)=2 c^{2} t \int_{-\psi_{x}}^{\psi_{\alpha}} \cos [B(c t, \psi)] \mathrm{d} \psi, \\
& K^{(\mathrm{b})}(c)=\int_{-\psi_{x}}^{\psi_{x}} C(c t, \psi) \mathrm{d} \psi+S\left(-\psi_{\alpha}\right)-S\left(\psi_{\alpha}\right), \\
& K^{(\mathrm{c})}(c)=\int_{-\psi_{x}}^{\psi_{\alpha}} D(c t, \psi) \mathrm{d} \psi+\int_{\psi_{x}}^{\pi-\psi_{\alpha}} D\left(\frac{L_{\min }}{\sin \psi}, \psi\right) \mathrm{d} \psi ;
\end{aligned}
$$

if $L_{\max } \leqslant c t<\sqrt{L_{1}^{2}+L_{3}^{2}}$,

$$
\begin{aligned}
& K^{(\mathrm{a})}(c)=2 c^{2} t\left\{\int_{-\psi_{x}}^{-\psi_{\beta}}+\int_{\psi_{f}}^{\psi_{z}}\right\} \cos [B(c t, \psi)] \mathrm{d} \psi \\
& K^{(\mathrm{b})}(c)=\left\{\int_{-\psi_{\alpha}}^{-\psi_{\beta}}+\int_{\psi_{\beta}}^{\psi_{\alpha}}\right\} C(c t, \psi) \mathrm{d} \psi+S\left(-\psi_{x}\right)-S\left(\psi_{\alpha}\right)+T\left(\psi_{\beta}\right)-T\left(-\psi_{\beta}\right), \\
& K^{(\mathrm{c})}(c)=\left\{\int_{-\psi_{x}}^{-\psi_{\beta}}+\int_{\psi_{\beta}}^{\psi_{\alpha}}\right\} D(c t, \psi) \mathrm{d} \psi+\int_{\psi_{x}}^{\pi-\psi_{\alpha}} D\left(\frac{L_{\min }}{\sin \psi}, \psi\right) \mathrm{d} \psi+\int_{-\psi_{\beta}}^{\psi_{\beta}} D\left(\frac{L_{\max }}{\cos \psi}, \psi\right) \mathrm{d} \psi ;
\end{aligned}
$$

if $\sqrt{L_{1}^{2}+L_{3}^{2}} \leqslant c t$,

$$
\begin{aligned}
& K^{(\mathrm{a})}(\mathrm{c})=0 \\
& K^{(\mathrm{b})}(c)=0 \\
& K^{(\mathrm{c})}(c)=\int_{\psi_{\gamma}}^{\pi_{-\psi_{\gamma}}} D\left(\frac{L_{\min }}{\sin \psi}, \psi\right) \mathrm{d} \psi+\int_{-\psi_{\gamma}}^{\psi_{\gamma}} D\left(\frac{L_{\max }}{\cos \psi}, \psi\right) \mathrm{d} \psi .
\end{aligned}
$$

The remaining integrals in (C.18)-(C.20) must be computed numerically. They are one-dimensional integrals with finite limits, to be compared with the two-dimensional integrals with infinite limits appearing in (48), with the restriction, however, that some of them involve the sine integral function. 